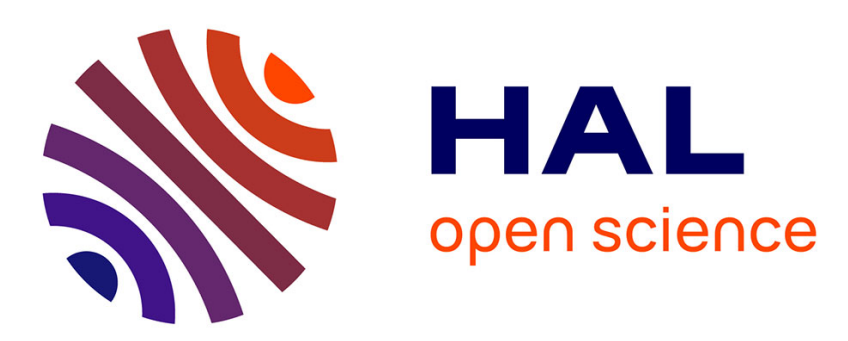

\title{
Nonlinear control with integral sliding properties for circular aerial robot trajectory tracking: Real-time validation
}

E. Ibarra-jimenez, Pedro Castillo Garcia, H. Abaunza

\section{- To cite this version:}

E. Ibarra-jimenez, Pedro Castillo Garcia, H. Abaunza. Nonlinear control with integral sliding properties for circular aerial robot trajectory tracking: Real-time validation. International Journal of Robust and Nonlinear Control, 2020, 30 (2), pp.609-635. 10.1002/rnc.4777 . hal-02445349

\author{
HAL Id: hal-02445349 \\ https://hal.science/hal-02445349
}

Submitted on 15 Feb 2021

HAL is a multi-disciplinary open access archive for the deposit and dissemination of scientific research documents, whether they are published or not. The documents may come from teaching and research institutions in France or abroad, or from public or private research centers.
L'archive ouverte pluridisciplinaire HAL, est destinée au dépôt et à la diffusion de documents scientifiques de niveau recherche, publiés ou non, émanant des établissements d'enseignement et de recherche français ou étrangers, des laboratoires publics ou privés. 


\section{Nonlinear control with integral sliding properties for circular aerial robot trajectory tracking: real-time validation}

\section{E. Ibarra-Jimenez ${ }^{1}$ | P. Castillo ${ }^{1 *}$ | H. Abaunza ${ }^{1}$}

${ }^{1}$ Sorbonne Universités, Université de Technologie de Compiégne, CNRS, UMR 7253 Heudiasyc, France.

\section{Correspondence}

P. Castillo,

Sorbonne Universités, Université de Technologie de Compiégne, CNRS, UMR 7253 Heudiasyc, France.

Email: castillo@hds.utc.fr

Funding information

This research was partially supported by Mexican CONACYT Project 262931. This work has been sponsored by the French government research program Investissements d'avenir through the Robotex Equipment of Excellence (ANR-10-EQPX-44)
A nonlinear control algorithm for tracking dynamic trajectories using an aerial vehicle is developed in this work. The control structure is designed using a sliding mode methodology which contains integral sliding properties. The stability analysis of the closed-loop system is proved using the Lyapunov formalism, ensuring convergence in a desired finite time and robustness towards unknown and external perturbations from the first time instant, even for high frequency disturbances. In addition, a dynamic trajectory is constructed with the translational dynamics of an aerial robot for autonomous take-off, surveillance missions and landing. This trajectory respects the constraints imposed by the vehicle characteristics allowing free initial trajectory conditions. Simulation results demonstrate the good performance of the controller in closed-loop system when a quadrotor follows the designed trajectory. In addition, flight tests are developed to validate the trajectory and the controller behavior in real time.

\section{KEYWORDS}

Robust and nonlinear control, integral sliding mode, aerial robots, path tracking, dynamic trajectory, real-time validation

Abbreviations: SMC, sliding mode control; ISM, integral sliding mode; MIMO, multiple input multiple output; UAV, unmanned aerial vehicle. 


\section{INTRODUCTION}

New civil applications for aerial robots are being developed every day. In recent years, novel implementations of these vehicles have been increasing in complexity, demanding a higher control performance to accomplish their missions. A recurrent task for Unmanned Aerial Vehicles (UAVs) is target inspection and surveillance, which requires that the aerial vehicle continuously tracks its objective with high precision and robustness. Quadrotors are a kind of UAV that has been widely studied in recent years due to their mechanical simplicity and broad availability, typically, these vehicles are capable of performing rotation and translation movements over three axes (six degrees of freedom). This configuration consists on four rotating blades whose aerodynamic effects result on three total torque components and one total thrust (four control inputs).

Quadrotor dynamics are often modeled as a nonlinear underactuated Multiple Input Multiple Output (MIMO) system. Controlling this kind of vehicles is a complex problem that requires effective methodologies to ensure system stability. A common practice to facilitate the development of quadrotor controllers (and other nonlinear MIMO systems) relies on linearizing the dynamic equations around equilibrium points or through defining linear models that approximate the nonlinear equations behavior under certain limited conditions, see [1] and [2].

Linearization techniques usually consider that only small variations occur in the internal system dynamics, in quadrotors this means that only small angles and slow velocities are taken into account in the model, converting the nonlinear problem in an easier linear or quasi-linear one in which the existing theory for linear control systems can be applied. For example in [3], a quadrotor was restricted such that only yaw and vertical movements could be performed, therefore reducing the control problem from a mathematical point of view. In [4], classical Proportional-IntegralDerivative (PID) and Linear-Quadratic (LQ) controllers were used to control the orientation of a quadrotor which was fixed on a 3D universal joint, these controllers were developed from a simplified linear version of the quadrotor dynamics. The main disadvantage of these approaches is that the resulting controllers are only valid locally, in a limited region around the equilibrium point. In quadrotors this means that the vehicle could become unstable at high angular inclinations or velocities, compromising the success of demanding missions.

In consequence, the study and development of controllers for nonlinear MIMO systems has been a subject of increasing interest in recent years. Some of the most popular approaches to solve this kind of problems are backstepping techniques like [5], [6], and [7], flatness algorithms as detailed in [8], [9], and [10] and Sliding Mode Controllers (SMC), see [11]-[15]. This last one has prorelyven to have an outstanding performance for controlling nonlinear systems by providing robustness properties, detailed in [16], and characteristics such as finite-time convergence as remarked in [17], and disturbance rejection as noted by [18].

In [15]-Chapter 4 an effective methodology is presented to control a certain type of MIMO nonlinear systems, here, the authors propose to transform the system into a regular form and then use a candidate Lyapunov function to solve the stability problem. Nevertheless, this methodology does not consider cases when the system is affected by parameters uncertainties or external disturbances. Therefore such controllers were not developed to achieve robustness.

It is well known that one of the advantages of sliding mode control is that it provides systems with robustness towards bounded parameter uncertainties and external disturbances (no mechanical system is subject to unbounded perturbations in the real world). However, in conventional sliding mode controllers, such robustness can only be guaranteed after the sliding surface converges to zero. This means that the disturbance compensation does not happen immediately after starting the system. A solution for better disturbance rejection and uncertainties compensation can be found on a variant of sliding mode algorithms proposed by [19] and [20], which is known as Integral Sliding Mode (ISM), this algorithm contains properties which can compensate bounded uncertainties since the beginning.

The application of sliding mode controllers for UAVs has been addressed by some works to provide system ro- 
bustness. For instance [21]-[23] used sliding mode observers to obtain state signals which were then introduced to the control loop. In [24], a super-twisting sliding mode controller with adaptive gains was developed for a UAV with 3 degrees of freedom. Other works like [25] and [26] have tackled the problem of compensating disturbances and uncertainties on quadrotors with adaptive sliding mode control schemes. Trajectory tracking algorithms based on sliding mode controllers have been proposed in [27] and by using multiple control layers. However, all of these works have limited the validation of their proposed algorithms to simulations (in most cases), or to constrained experimental setups, see [28] and [29].

The main scope of this work is the development of an integral sliding mode control algorithm to stabilize a quadrotor modeled as a nonlinear MIMO system. The algorithm is composed by two parts, the first one is designed to compensate external perturbations since the beginning, while the second one consists on a super-twisting sliding mode controller which ensures the convergence of a sliding surface to zero in a desired finite time. As consequence, our proposal provides fast stabilization of the system dynamics without increasing chattering effect, see [30], [31].

Therefore, the main contributions of this work are:

1. The design of a robust nonlinear controller to stabilize a quadrotor in presence of unknown external disturbances with high frequency.

2. The proposed controllers compensate bounded uncertainties from the beginning, guaranteeing a faster stabilization.

3. The conception of a trajectory generation algorithm for quadrotors, with dynamic properties for takeoff, landing, and surveillance missions.

4. Real-time validation of the trajectory generation and control schemes in autonomous flight tests.

The rest of the paper is organized as follows: In Section 2 the preliminaries for modeling the nonlinear quadrotor dynamic model, as well as the mathematical preliminaries of the trajectory generation algorithm are introduced. In Section 3 the circular dynamic trajectory design is presented, detailing its autonomous takeoff, target tracking and landing properties based on Hopf bifurcations. The design of a nonlinear integral sliding mode controller for robust and fast trajectory tracking is detailed in Section 4. Numerical simulations of the proposed algorithms are depicted in Section 5. In addition, flight experiments are presented in Section 6, that validate the proposed schemes in real time. Finally, conclusions about this work and future improvements are discussed in Section 7.

\section{| PROBLEM STATEMENT}

Quadrotors are commonly used for inspection, surveillance and target tracking missions. In this kind of tasks it is often necessary to expand the aerial vehicle workspace for reaching a wider area for inspection, or in the case of target tracking, increasing its vision angle for relocating a lost or hidden target. If the objective to be surveilled is static, the problem is relatively easy to solve using classical approaches. The challenge arises with a moving target performing random displacements, in this case the controller should be robust enough to ensure tracking without loosing the target.

In this paper, we propose a solution based on a nonlinear sliding-mode controller for trajectory tracking. This solution also includes the conception of a time-varying trajectory that adapts its dynamics with respect to target movements. The control law will be designed to compensate unknown external perturbations from the beginning and to converge the sliding surfaces to zero in a desired finite time.

The scenario for validating this approach is the following: A quadrotor takes-off autonomously from a random position either far or close from its target until a desired altitude is reached, while the vehicle is lifting, its objective 
will be to describe circles such that its center is located over the target at a constant height (see Figure 1). If the target moves, the circular trajectory will be adapted and reconstructed to fit its behavior. And finally, if a remote operator indicates the mission ending, the drone will autonomously land accordingly.

\section{| Model background and trajectory design preliminaries}

The quadrotor is an under-actuated system with fast and unstable nonlinear dynamics such that any failure in the closed-loop system may cause the vehicle to crash. Its nonlinear model is commonly described as

$$
\begin{aligned}
\ddot{x} & =-\sin (\theta) \frac{1}{m} U_{1} \\
\ddot{y} & =\cos (\theta) \sin (\phi) \frac{1}{m} U_{1} \\
\ddot{z} & =\cos (\theta) \cos (\phi) \frac{1}{m} U_{1}-g \\
\ddot{\phi} & =\dot{\theta} \dot{\psi}\left(\frac{I_{y}-I_{x}}{I_{x}}\right)-\frac{I_{r}}{I_{x}} \dot{\theta} \Omega+\frac{1}{I_{x}} U_{\phi}+\xi_{1} \\
\ddot{\theta} & =\dot{\phi} \dot{\psi}\left(\frac{I_{z}-I_{x}}{I_{y}}\right)-\frac{I_{r}}{I_{y}} \dot{\phi} \Omega+\frac{1}{I_{y}} U_{\theta}+\xi_{2} \\
\ddot{\psi} & =\dot{\theta} \dot{\phi}\left(\frac{I_{x}-I_{y}}{I_{z}}\right)+\frac{1}{I_{z}} U_{\psi}+\xi_{3}
\end{aligned}
$$

where the position coordinates are defined as $x, y, z$ while its orientation is represented by Euler angles; roll $(\phi)$, pitch $(\theta)$ and yaw. $(\psi)$ The angular acelerations of the body frame are considered to be aligned with the inertial frame in this model. The distance between each motor to the vehicle gravitational center is denoted by $/$. The body inertia over each axis is defined by $I_{x}, I_{y}$ and $I_{z}$, while the inertia of each motor is represented by $I_{r}$. $U_{i}$ symbolizes the control input, the rotor speed is defined by $\Omega$ and $\xi_{i}$ represents external and unknown disturbances, see Figure 1.

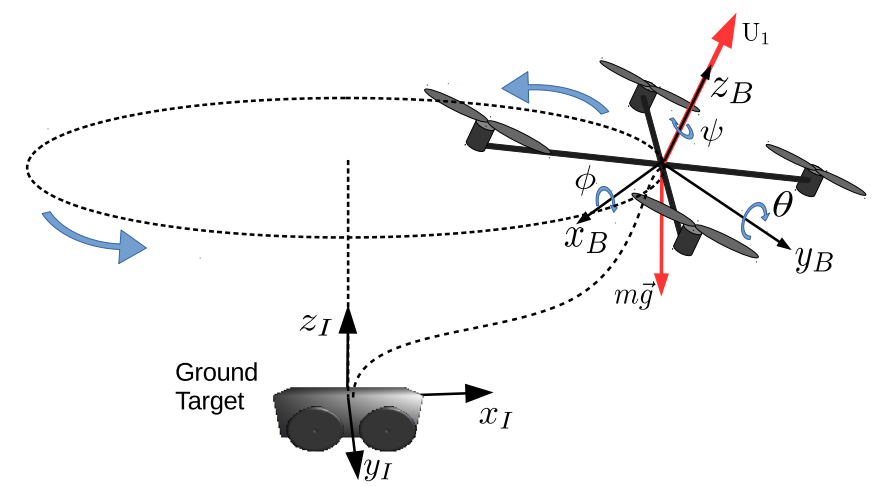

FIGURE 1 A quadrotor surveying a ground target (moving at a constant velocity) with a circular trajectory. The inertial reference frame, located at the target center, is denoted as $\mathcal{I}=\left\{x_{I}, y_{I}, z_{I}\right\}$, while the body frame is represented as $\mathcal{B}=\left\{x_{B}, y_{B}, z_{B}\right\}$.

One of the objectives of this work is to design a 3D dynamic trajectory that will be followed by the system described by (1)-(6). The goal of this trajectory is to track a ground target even if it moves with random movements.

Some ideas from [32] are taken to conceive the trajectory. Define a circular relation as $r^{2}=x_{r}^{2}+y_{r}^{2}$ and $\tan (\lambda)=\frac{y_{r}}{x_{r}}$, where $r$ denotes the desired trajectory radio and $x_{r}$ and $y_{r}$ are its position coordinates in the horizontal $x, y$ plane. 
From the previous, a nonlinear system can be stated as

$$
\begin{aligned}
& \dot{x}_{r}=\mu_{1} x_{r}+\gamma_{1} y_{r}-x_{r}\left(x_{r}^{2}+y_{r}^{2}\right) \\
& \dot{y}_{r}=-\gamma_{1} x_{r}+\mu_{1} y_{r}-y_{r}\left(x_{r}^{2}+y_{r}^{2}\right)
\end{aligned}
$$

where $\gamma_{1}$ and $\mu_{1}$ are bifurcation parameters. Notice that system (7) has a single equilibrium point at the origin. Observe that for certain values of $\mu_{1}$, the dynamics $\left(x_{r}, y_{r}\right)$ will perform differently around the equilibrium point $(0,0)$ as seen in Figure 2.
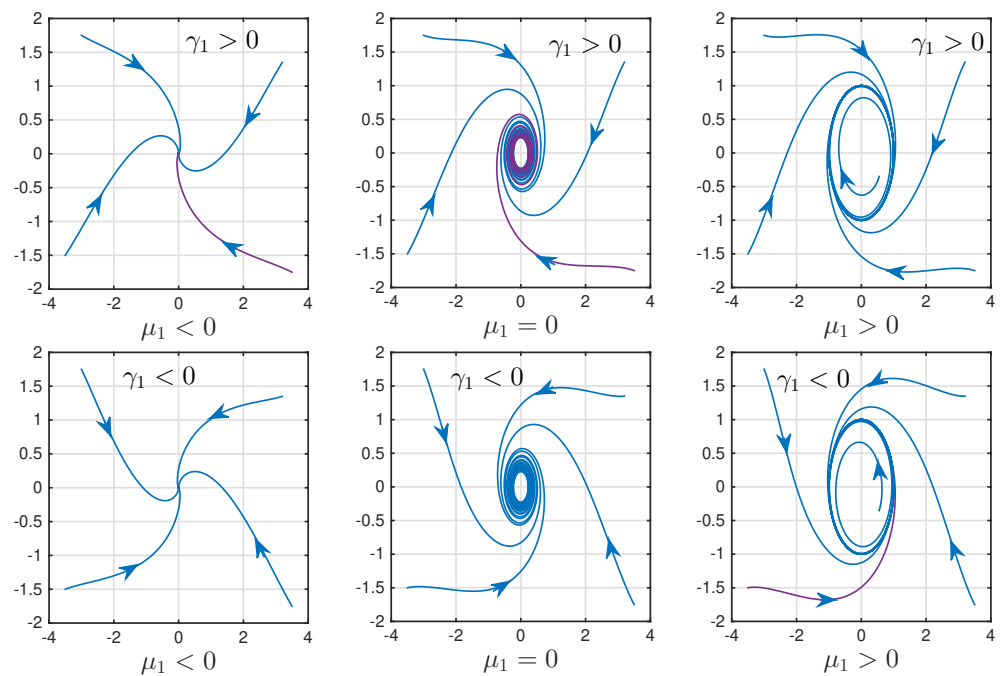

FIG URE 2 Development of a limit cycle in a Hopf bifurcation

From (7), the corresponding polar coordinates can be represented by

$$
\dot{r}=r\left(\mu_{1}-r^{2}\right), \quad \dot{\lambda}=-\gamma_{1}
$$

Observe from Figure 2 the different stability cases for parameters $\mu_{1}<0, \mu_{1}=0$ and $\mu_{1}>0$, with $\gamma_{1}>0$ and $\gamma_{1}<0$ respectively. If $\mu_{1} \leq 0$ then the entire diagram consists on a stable spiral at the origin. If $\mu_{1}>0$ then there is an unstable spiral at the origin surrounded by a stable limit cycle which grows out of the origin. Remark that the parameter $\mu_{1}$ generates a limit cycle and then the Hopf bifurcation appears.

In addition, $\gamma_{1}$ produces a steady clockwise spiral motion if $\gamma_{1}>0$, which becomes counterclockwise if $\gamma_{1}<0$. Finally, note from (8) that when the bifurcation parameter $\mu_{1}=r^{2}>0$ then $r=\sqrt{\mu_{1}}$.

It is straightforwardly deduced that (7) can yield a dynamic trajectory in the horizontal plane, nevertheless, a 3-dimensional movement is desired in this work. Imposing a constant altitude $h$ for the quadrotor and defining a vertical error as $e_{z_{r}}=z_{r}-h$, it follows that $\dot{e}_{z_{r}}=\dot{z}_{r}$, where $z_{r}$ denotes the reference height.

For ensuring asymptotic converge for $e_{z}$, propose $\dot{e}_{z_{r}}=-k e_{z_{r}}$ with a constant $k>0$. Therefore, the trajectory dynamics for the $z$ coordinate can be defined as

$$
\dot{z}_{r}=-k\left(z_{r}-h\right)
$$


Consider that the aerial vehicle will take-off from an arbitrary horizontal position $x, y$ with $z=0$, thus, the initial position for the trajectory can be defined as $x_{r}(0)=x_{r_{0}}, y_{r}(0)=y_{r_{0}}, z_{r}(0)=0$. From (7) the initial conditions for the velocity become $\dot{x}_{r}(0)=\dot{x}_{r_{0}}, \dot{y}_{r}(0)=\dot{y}_{r_{0}}$ and $\dot{z}_{r}(0)=\dot{z}_{r_{0}}$, which yield:

$$
\begin{aligned}
& \dot{x}_{r_{0}}=\mu_{1} x_{r_{0}}+\gamma_{1} y_{r_{0}}-x_{r_{0}}\left(x_{r_{0}}^{2}+y_{r_{0}}^{2}\right), \\
& \dot{y}_{r_{0}}=-\gamma_{1} x_{r_{0}}+\mu_{1} y_{r_{0}}-y_{r_{0}}\left(x_{r_{0}}^{2}+y_{r_{0}}^{2}\right), \\
& \dot{z}_{r_{0}}=k h .
\end{aligned}
$$

Notice that the previous equations were obtained using classical approaches, implying that high values on the initial velocity of the vehicle could yield strong responses from many controllers, often resulting in aggressive maneuvers that could damage the quadrotor in real-world implementations.

To overcome this problem, a solution will be proposed in which the initial velocity conditions can be chosen freely without compromising the system robustness.

\section{D DYNAMIC TRAJECTORY}

Propose the following functions

$$
\begin{aligned}
& S_{x}=x_{2_{r}}-\mu_{1} x_{1_{r}}-\gamma_{1} y_{1_{r}}+x_{1_{r}}\left(x_{1_{r}}^{2}+y_{1_{r}}^{2}\right) \\
& S_{y}=y_{2_{r}}+\gamma_{1} x_{1_{r}}-\mu_{1} y_{1_{r}}+y_{1_{r}}\left(x_{1_{r}}^{2}+y_{1_{r}}^{2}\right) \\
& S_{z}=z_{2_{r}}+k\left(z_{1_{r}}-h\right)
\end{aligned}
$$

Notice that, if $S_{i} \rightarrow 0$, then systems (7) and (9) are obtained for $x_{1_{r}}=x_{r}, x_{2_{r}}=\dot{x}_{r}, y_{1_{r}}=y_{r}, y_{2_{r}}=\dot{y}_{r}, z_{1_{r}}=z_{r}$ and $z_{2_{r}}=\dot{z}_{r}$.

\section{| Take-Off stage of movement}

Define

$$
\begin{array}{lll}
\dot{x}_{1_{r}}=x_{2_{r}} ; & \dot{y}_{1_{r}}=y_{2_{r}} ; \quad \dot{z}_{1_{r}}=z_{2_{r}}, \\
\dot{x}_{2_{r}}=u_{x} ; & \dot{y}_{2_{r}}=u_{y} ; \quad \dot{z}_{2_{r}}=u_{z},
\end{array}
$$

such that

$$
\begin{aligned}
& u_{x}=-\left(3 x_{1_{r}}^{2}+y_{1_{r}}^{2}-\mu_{1}\right) x_{2_{r}}-\left(2 x_{1_{r}} y_{1_{r}}-\gamma_{1}\right) y_{2_{r}}-k_{x} S_{x}, \\
& u_{y}=-\left(2 x_{1_{r}} y_{1_{r}}+\gamma_{1}\right) x_{2 r}-\left(3 y_{1_{r}}^{2}+x_{1_{r}}^{2}-\mu_{1}\right) y_{2_{r}}-k_{y} S_{y}, \\
& u_{z}=-k z_{2 r}-k_{z} S_{z},
\end{aligned}
$$

with $k_{x}, k_{y}, k_{z}>0$.

Taking the derivative of (11) and using the previous equations, it follows that

$$
\dot{S}_{x}=-k_{x} S_{x} ; \dot{S}_{y}=-k_{y} S_{y} ; \dot{S}_{z}=-k_{z} S_{z}
$$

whose solutions are $S_{x}(t)=e^{-k_{x} t} S_{x}(0) ; S_{y}(t)=e^{-k_{y} t} S_{y}(0)$ and $S_{z}(t)=e^{-k_{z} t} S_{z}(0)$. 
This implies that (11) can be written as

$$
\begin{aligned}
& x_{2_{r}}=\mu_{1} x_{1_{r}}+\gamma_{1} y_{1_{r}}-x_{1_{r}}\left(x_{1_{r}}^{2}+y_{1_{r}}^{2}\right)+e^{-k_{x} t} S_{x}(0), \\
& y_{2_{r}}=-\gamma_{1} x_{1_{r}}+\mu_{1} y_{1_{r}}-y_{1_{r}}\left(x_{1_{r}}^{2}+y_{1_{r}}^{2}\right)+e^{-k_{y} t} S_{y}(0), \\
& z_{2_{r}}=-k\left(z_{1_{r}}-h\right)+e^{-k_{z} t} S_{z}(0),
\end{aligned}
$$

and finally,

$$
\begin{aligned}
& x_{2_{r_{0}}}=\underbrace{\mu_{1} x_{1_{r_{0}}}+\gamma_{1} y_{1_{r_{0}}}-x_{1_{r_{0}}}\left(x_{1_{r_{0}}}^{2}+y_{1_{r_{0}}}^{2}\right)}_{x_{2_{r}}(0)-S_{x}(0)}+S_{x}(0), \\
& y_{2_{r_{0}}}=\underbrace{-\gamma_{1} x_{1_{r_{0}}}+\mu_{1} y_{1_{r_{0}}}-y_{1_{r_{0}}}\left(x_{1_{r_{0}}}^{2}+y_{1_{r_{0}}}^{2}\right)}_{y_{2_{r}}(0)-S_{y}(0)}+S_{y}(0), \\
& z_{2_{r_{0}}}=\underbrace{-k\left(z_{1_{r_{0}}}-h\right)}_{z_{2_{r}}(0)-S_{z}(0)}+S_{z}(0),
\end{aligned}
$$

Observe from above that $x_{2_{0}}, y_{2_{r_{0}}}$, and $z_{2_{r_{0}}}$ are independent, and can be chosen freely.

Equation (12) defines the first stage for reaching a desired altitude $h$. This system was simulated in the time interval $0 \leq t<T_{F 1}$ with $T_{F 1}=15 \mathrm{~s}$, choosing initial conditions $x_{1_{r}}(0)=1.5, y_{1_{r}}(0)=-3, z_{1_{r}}(0)=0$ in meters and $x_{2_{r}}(0)=y_{2_{r}}(0)=z_{2_{r}}(0)=0 \mathrm{in} \mathrm{m} / \mathrm{s}$, with $h=2 \mathrm{~m}$. The constant parameters were selected as $\mu_{1}=4, \gamma_{1}=1$, and $k=k_{x}=k_{y}=k_{z}=1$. The trajectory performance is depicted in Figure 3 .

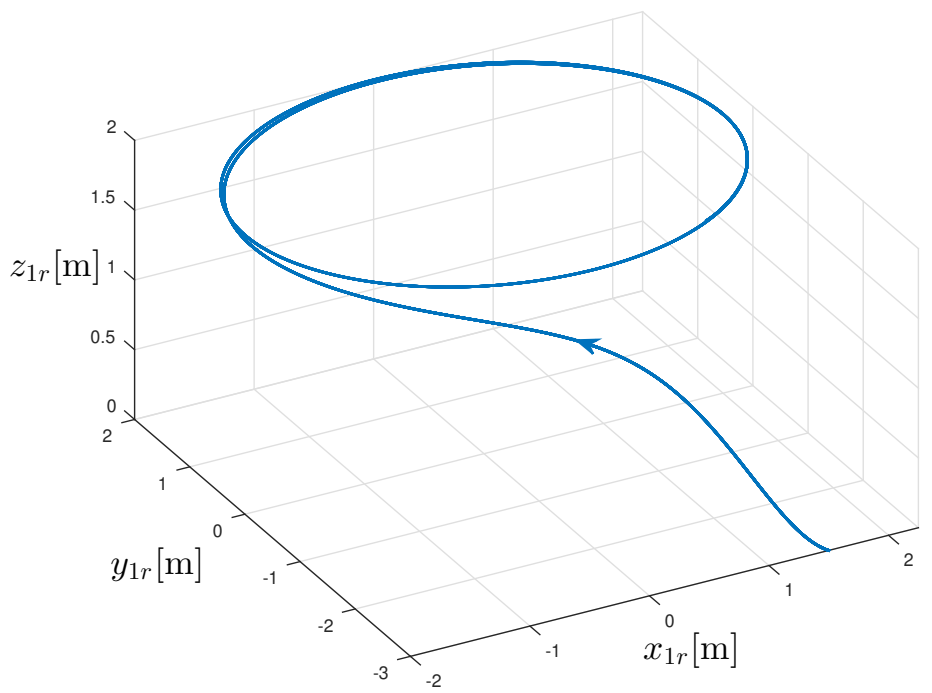

FIGURE 3 Dynamic circular trajectory to take-off and reach a desired altitude $h$.

The behavior of the sliding surfaces $S_{x}, S_{y}$ and $S_{z}$ is illustrated in Figure 4, note that these functions converge asymptotically to zero guaranteeing a smooth convergence to the desired altitude. 

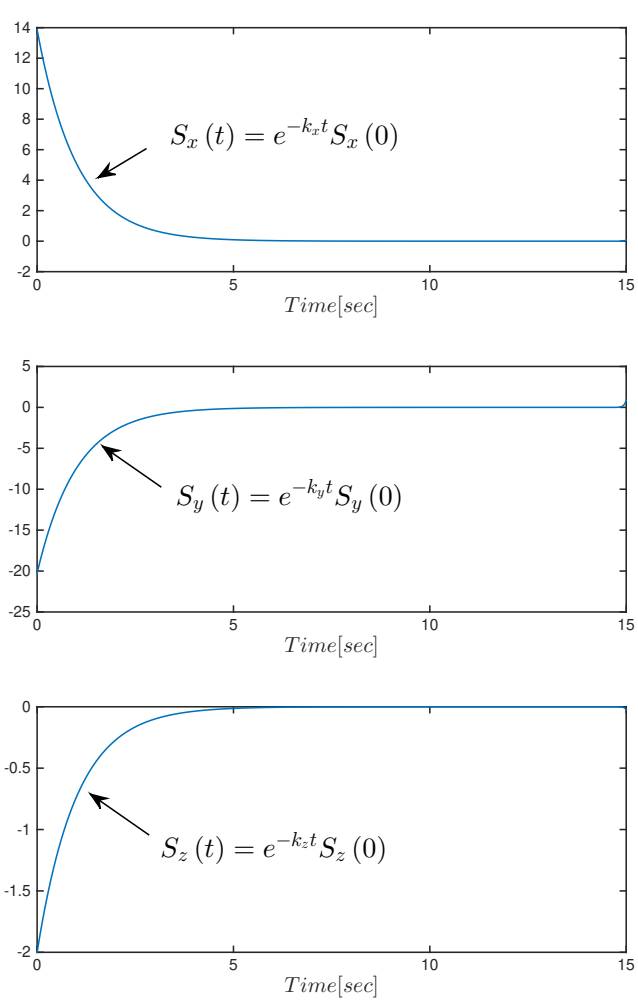

FIGURE 4 Performance of the sliding surfaces $S_{x}, S_{y}$ and $S_{z}$.

\section{| Circular and landing stages of movements}

Once the desired altitude is reached, two possible scenarios may arrive $\forall t \geq T_{F 1}$ :

a.- Landing to the point $\left(0,0, z_{1_{r_{0}}}\right)$ at $T_{F 1} \leq t \leq T_{F}$. This implies that the aerial vehicle will start the asymptotic landing at time $T_{F 1}$, ending at $T_{F}$.

b.- Spiral surveillance at $T_{F 1} \leq t<T_{F 2}$ around $(0,0, h)^{1}$, and landing to $\left(0,0, z_{1_{r_{0}}}\right)$ at $T_{F 2} \leq t \leq T_{F}$.

The complete 3D dynamic trajectory is represented in the following theorem.

Theorem 1 A 3D dynamic trajectory, $\ddot{\xi}_{r}$, with properties of take-off, circular motion and landing can be defined as

$$
\ddot{\xi}_{r}=\mathbf{g}(t) \mathbf{u}+\mathbf{f}(t) \mathbf{v},
$$

with $\xi_{r}=\left[x_{r}, y_{r}, z_{r}\right], \mathbf{g}(t)=[\tilde{g}(t), \tilde{g}(t), \bar{g}(t)], \mathbf{u}=\left[u_{x}, u_{y}, u_{z}\right], \mathbf{f}(t)=[\tilde{f}(t), \tilde{f}(t), \bar{f}(t)], \mathbf{v}=\left[v_{x}, v_{y}, v_{z}\right]$.

\footnotetext{
${ }^{1}$ For simplifying the procedure, the origin in the plane $x, y$ is considered as the center of the trajectory, nevertheless, these coordinates could be changed.
} 
In the scalar form,

$$
\begin{array}{ll}
\dot{x}_{1_{r}}=x_{2_{r}}, & \dot{x}_{2_{r}}=\tilde{g}(t) u_{x}+\tilde{f}(t) v_{x}, \\
\dot{y}_{1_{r}}=y_{2_{r}}, & \dot{y}_{2_{r}}=\tilde{g}(t) u_{y}+\tilde{f}(t) v_{y}, \\
\dot{z}_{1_{r}}=z_{2_{r}}, & \dot{z}_{2_{r}}=\bar{g}(t) u_{z}+\bar{f}(t) v_{z},
\end{array}
$$

where $\mathbf{u}$ is defined in (13) and $\mathbf{v}$ is proposed as

$$
\begin{aligned}
& v_{x}=-\left(3 x_{1_{r}}^{2}+y_{1_{r}}^{2}-\mu_{2}\right) x_{2 r}-\left(2 x_{1_{r}} y_{1_{r}}-\gamma_{2}\right) y_{2_{r}}-\bar{k}_{x} \sigma_{x} \\
& v_{y}=-\left(2 x_{1_{r}} y_{1_{r}}+\gamma_{2}\right) x_{2_{r}}-\left(3 y_{1_{r}}^{2}+x_{1_{r}}^{2}-\mu_{2}\right) y_{2_{r}}-\bar{k}_{y} \sigma_{y} \\
& v_{z}=-\bar{k} z_{2_{r}}-\bar{k}_{z} \sigma_{z}
\end{aligned}
$$

with

$$
\begin{aligned}
\sigma_{x} & =\dot{x}_{1_{r}}-\mu_{2} x_{1_{r}}-\gamma_{2} y_{21_{r}}+x_{1_{r}}\left(x_{1_{r}}^{2}+y_{1_{r}}^{2}\right), \\
\sigma_{y} & =\dot{y}_{1_{r}}+\gamma_{2} x_{1_{r}}-\mu_{2} y_{1_{r}}+y_{1_{r}}\left(x_{1_{r}}^{2}+y_{1_{r}}^{2}\right), \\
\sigma_{z} & =\dot{z}_{1_{r}}+\bar{k}\left(z_{1_{r}}-z_{1_{r}}(0)\right) .
\end{aligned}
$$

where the bifurcation parameters must be selected as $\mu_{1}=r^{2}>0$ with $\left(\gamma_{1}>0\right.$ or $\left.\gamma_{1}<0\right)$ and $\mu_{2} \leq 0$ with $\left(\gamma_{2}>0\right.$ or $\left.\gamma_{2}<0\right)$.

$\tilde{f}(t)$ and $\bar{f}(t)$ are designed as

$$
\begin{aligned}
& \tilde{f}(t)=\left\{\begin{array}{cc}
e^{\left(n\left(t-T_{F 1}\right)\right)} & 0 \leq t<T_{F 1} \\
1 & T_{F 1} \leq t \leq T_{F} \\
e^{\left(n\left(t-T_{F 2}\right)\right)} & 0 \leq t<T_{F 2} \\
1 & T_{F 2} \leq t \leq T_{F}
\end{array} \mid n>0\right.
\end{aligned}
$$

where $T_{F 1} \leq T_{F 2}<T_{F}$.

Finally, $\tilde{g}(t)=1-\tilde{f}(t)$ and $\bar{g}(t)=1-\bar{f}(t)$.

In addition, these trajectory properties imply that the initial velocities are independent and can be chosen freely.

The proof of the theorem is given in Appendix A.

The trajectory with both scenarios for landing was verified numerically and illustrated in Figures 5 and 6 . The simulation values are written in Table 1.

\begin{tabular}{|c|c|c|c|c|c|}
\hline$n=10$ & $\mu_{1}=4$ & $\mu_{2}=-0.25$ & $T_{F 1}=15 s$ & $\gamma_{1}=1$ & $\gamma_{2}=-1$ \\
\hline$T_{F 2}=35 s$ & $k=1$ & $\bar{k}=0.5$ & $T_{F}=50 s$ & $k_{x}=1$ & $\bar{k}_{x}=2$ \\
\hline$h=2$ & $k_{y}=1$ & $\bar{k}_{y}=2$ & $k_{z}=1$ & $\bar{k}_{z}=0.5$ & \\
\hline
\end{tabular}

TAB LE 1 Constant parameters used for simulation purposes.

In Figure 5, observe the evolution of the dynamic trajectory with two stages of movement: take-off and landing. In 
contrast, Figure 6 displays the complete scenario: take-off, spiral motion and landing.

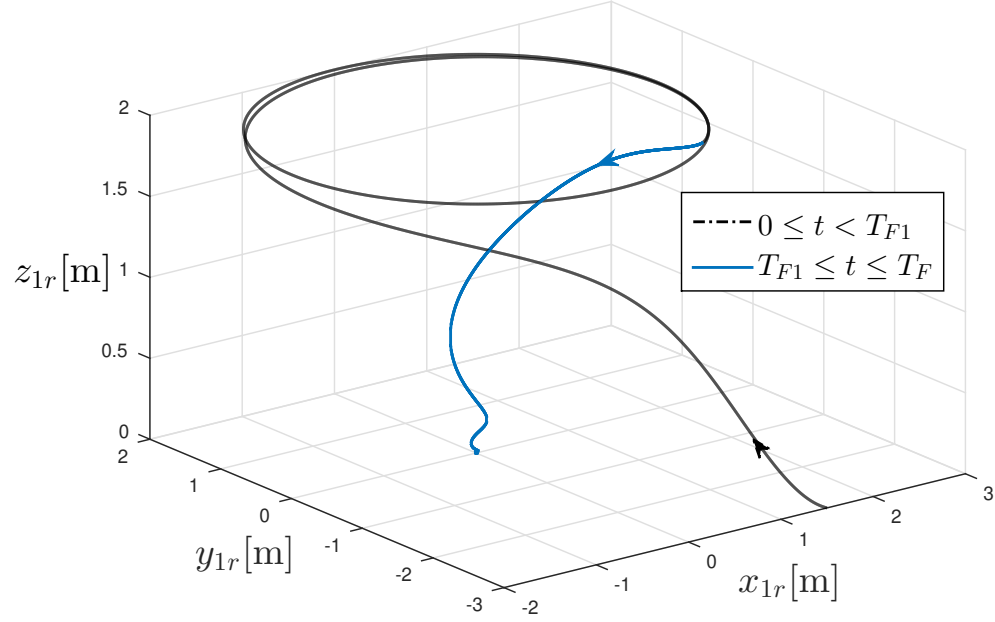

FIGURE 5 Trajectory performance for take-off to a desired altitude $h$ and landing to $\left(0,0, z_{1_{r}}(0)\right)$.

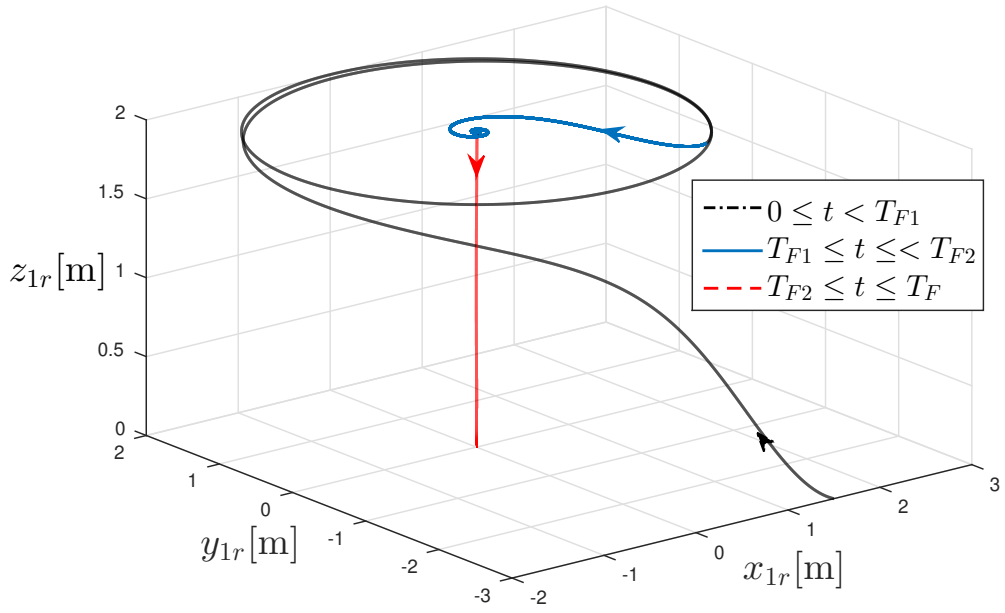

FIGURE 6 Trajectory behavior for the full scenario; take-off, spiral motion around $(0,0, h)$ and landing to $\left(0,0, z_{1_{r}}(0)\right)$.

\section{NONLINEAR CONTROL FOR TRACKING A DYNAMIC TRAJECTORY}

The challenge in this section is to design a robust nonlinear controller for tracking a desired trajectory. It will be constructed for compensating unknown and external disturbances.

From (1)-(6), the nonlinear dynamics of the quadrotor can be separated in two subsystems for the translational and rotational movements, which will be controlled by following the scheme illustrated in Figure 7. Due the system characteristics (under-actuated) the controller will be proposed for high and low sequence levels giving more priority to the low level (attitude) control. In the following, the path tracking equations are developed. 


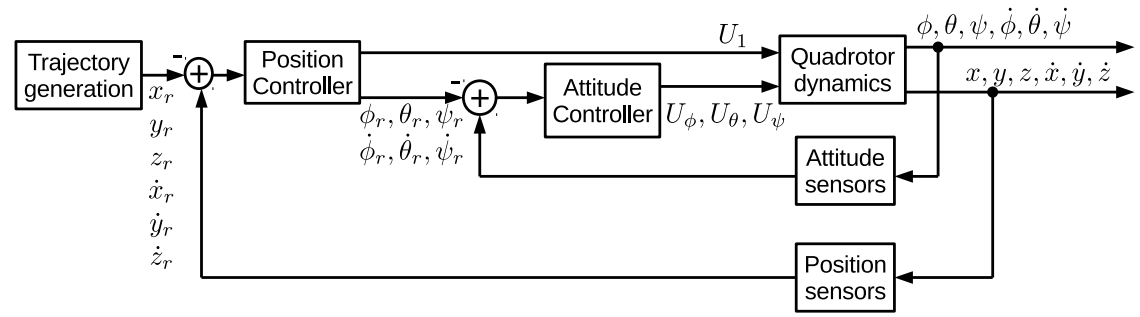

FIGURE 7 Quadrotor control diagram, including trajectory generation, attitude and position control algorithms.

\section{| Translational tracking}

In order to track the desired 3D trajectory, define the following errors for the position $e_{x_{1}}=x_{1}-x_{1_{r}}, e_{y_{1}}=y_{1}-y_{1_{r}}$, $e_{z_{1}}=z_{1}-z_{1_{r}}$, and for the velocity; $e_{x_{2}}=x_{2}-x_{2_{r}}, e_{y_{2}}=y_{2}-y_{2_{r}}, e_{z_{2}}=z_{2}-z_{2_{r}}$, where from (1)-(3), it follows that $x_{1}=x, y_{1}=y, z_{1}=z, x_{2}=\dot{x}, y_{2}=\dot{y}, z_{2}=\dot{z}, \phi_{1}=\phi, \theta_{1}=\theta, \psi_{1}=\psi$. The states with subscript $r$ define the desired trajectory, in this case it is defined by Theorem 1 .

Then, differentiating the position and velocity errors it yields

$$
\begin{array}{lll}
\dot{e}_{x_{1}}=e_{x_{2}}, & \dot{e}_{y_{1}}=e_{y_{2}}, & \dot{e}_{z_{1}}=e_{z_{2}}, \\
\dot{e}_{x_{2}}=\dot{x}_{2}-\dot{x}_{2_{r}}, & \dot{e}_{y_{2}}=\dot{y}_{2}-\dot{y}_{2_{r}}, & \dot{e}_{z_{2}}=\dot{z}_{2}-\dot{z}_{2_{r}} .
\end{array}
$$

Propose $U_{1}$ as the main thrust of the vehicle, such that its direction depends from the desired angles $\phi_{1}$, and $\theta_{1_{r}}$ as

$$
U_{1}=\frac{m\left[\dot{z}_{r_{2}}+g+V_{z}\right]}{\cos \left(\theta_{1_{r}}\right) \cos \left(\phi_{1_{r}}\right)},
$$

where $V_{z}$ will be defined later for ensuring asymptotic converge of the vertical dynamics. Define $\delta_{z}=\dot{z}_{r_{2}}+g$ and introducing $U_{1}$ into $\dot{e}_{z_{2}}$ in (18), it yields

$$
\dot{e}_{z_{2}}=\cos \left(\theta_{1}\right) \cos \left(\phi_{1}\right) \frac{1}{\not h}\left\{\frac{\not h}{\cos \left(\theta_{1_{r}}\right) \cos \left(\phi_{1_{r}}\right)}\left[\delta_{z}+V_{z}\right]\right\}-\delta_{z} .
$$

Assumption 4.1 An attitude controller given by $U_{\phi}, U_{\theta}, U_{\psi}$ exists such that the quadrotor angles reach a desired reference as $\phi_{1} \rightarrow \phi_{1_{r}}, \theta_{1} \rightarrow \theta_{1_{r}}, \psi_{1} \rightarrow \psi_{1_{r}}$ such that $\phi_{1_{r}}, \theta_{1_{r}} \neq 90^{\circ}$. This controller will be developed in Section 4.3.

Considering that Assumption 4.1 is true, it implies that $\dot{e}_{z_{1}}=e_{z_{2}}, \dot{e}_{z_{2}}=V_{z} \cdot V_{z}=-K_{p_{z}} e_{z_{1}}-K_{d_{z}} e_{z_{2}}$ can be proposed with $K_{p_{z}}, K_{d_{z}}>0$ to ensure asymptotic convergence of $e_{z_{1}}$ and $e_{z_{2}}$ around $(0,0)$.

The desired angles from (19) should also be dependent on the position reference in the horizontal plane $x, y$ for tracking trajectories, therefore, it is necessary to define them with respect to the $e_{x}$ and $e_{y}$ dynamics.

Introducing $U_{1}$ into $\dot{e}_{y_{2}}$, it follows that

$$
\dot{e}_{y_{2}}=\cos \left(\theta_{1}\right) \sin \left(\phi_{1}\right) \frac{1}{\not h t}\left\{\frac{\not \mu r}{\cos \left(\theta_{1_{r}}\right) \cos \left(\phi_{1_{r}}\right)}\left[\delta_{z}+V_{z}\right]\right\}-\dot{y}_{2_{r}} .
$$

Considering Assumption 4.1, then

$$
\dot{e}_{y_{2}}=\sin \left(\phi_{1_{r}}\right)\left\{\frac{1}{\cos \left(\phi_{1_{r}}\right)}\left[\delta_{z}+V_{z}\right]\right\}-\dot{y}_{2_{r}}=\tan \left(\phi_{1_{r}}\right)\left[\delta_{z}+V_{z}\right]-\dot{y}_{2_{r}} .
$$

Proposing

$$
\phi_{1_{r}}=\tan ^{-1}\left(\frac{\dot{y}_{2_{r}}+V_{y}}{\delta_{z}+V_{z}}\right)
$$


where $V_{y}$ will be defined later for assuring asymptotic converge. The previous implies that

$$
\dot{e}_{y_{1}}=e_{y_{2}}, \dot{e}_{y_{2}}=V_{y} .
$$

Define $V_{y}=-K_{p_{y}} e_{y_{1}}-K_{d_{y}} e_{y_{2}}$ with $K_{p_{y}}, K_{d_{y}}>0$, to achieve asymptotic convergence of $e_{y_{1}}$ and $e_{y_{2}}$ around $(0,0)$.

Introducing $U_{1}$ into $\dot{e}_{x_{2}}$ in (18), and following Assumption 4.1, it yields

$$
\dot{e}_{x_{2}}=-\tan \left(\theta_{1_{r}}\right)\left\{\frac{1}{\cos \left(\phi_{1 r}\right)}\left[\delta_{z}+V_{z}\right]\right\}-\dot{x}_{2_{r}}
$$

Propose

$$
\theta_{1_{r}}=\tan ^{-1}\left\{(-1) \cos \left(\phi_{1_{r}}\right)\left(\frac{\dot{x}_{2 r}+V_{x}}{\delta_{z}+V_{z}}\right)\right\}
$$

where $V_{x}$ will be obtained by introducing (23) into (22), which implies that $\dot{e}_{x_{1}}=e_{x_{2}}, \dot{e}_{x_{2}}=V_{x}$, defining $V_{x}=-K_{p_{x}} e_{x_{1}}-$ $K_{d_{x}} e_{x_{2}}$ with $K_{p_{x}}, K_{d_{x}}>0$, then the asymptotic convergence of $e_{x_{1}}$ and $e_{x_{2}}$ around $(0,0)$ will be achieved.

From (21) and (23), the desired angular velocities are represented by

$$
\begin{aligned}
\phi_{2 r} & =\frac{\cos ^{2}\left(\phi_{1_{r}}\right)}{r_{z}}\left[\dot{r}_{y}-\dot{r}_{z} \tan \left(\phi_{1_{r}}\right)\right], \\
\theta_{2 r} & =\frac{\cos ^{2}\left(\theta_{1_{r}}\right)}{r_{z}}\left[r_{x} \sin \left(\phi_{1_{r}}\right) \phi_{2_{r}}-\dot{r}_{z} \tan \left(\theta_{1_{r}}\right)-\dot{r}_{x} \cos \left(\phi_{1_{r}}\right)\right],
\end{aligned}
$$

with $r_{x}=\dot{x}_{2_{r}}+V_{x} ; r_{y}=\dot{y}_{2_{r}}+V_{y}$ and $r_{z}=\delta_{z}+V_{z}$. In addition,

$$
\begin{aligned}
\ddot{x}_{2 r} & =\tilde{f}(t)\left[\left(\dot{v}_{x}-\dot{u}_{x}\right)+n\left(v_{x}-u_{x}\right)\right]+\dot{u}_{x}, \\
\ddot{y}_{2 r} & =\tilde{f}(t)\left[\left(\dot{v}_{y}-\dot{u}_{y}\right)+n\left(v_{y}-u_{y}\right)\right]+\dot{u}_{y}, \\
\dot{\delta}_{z} & =\tilde{f}(t)\left[\left(\dot{v}_{z}-\dot{u}_{z}\right)+n\left(v_{z}-u_{z}\right)\right]+\dot{u}_{z},
\end{aligned}
$$

such that $\dot{V}_{x}=-K_{p_{x}} e_{x_{2}}-K_{d_{x}} \dot{e}_{x_{2}}, \dot{V}_{y}=-K_{p_{y}} e_{y_{2}}-K_{d_{y}} \dot{e}_{y_{2}}$, and $\dot{V}_{z}=-K_{p_{z}} e_{z_{2}}-K_{d_{z}} \dot{e}_{z_{2}}$. Therefore

$$
\begin{aligned}
& \dot{u}_{x}=-\left(3 x_{1_{r}}^{2}+y_{1_{r}}^{2}-\mu_{1}\right)\left(\dot{x}_{2 r}+k_{x} x_{2_{r}}\right)-\left(2 x_{1_{r}} y_{1_{r}}-\gamma_{1}\right)\left(\dot{y}_{2_{r}}+k_{x} y_{2_{r}}\right)-2 x_{1_{r}}\left(y_{2_{r}}^{2}+3 x_{2_{r}}^{2}\right)-4 x_{2_{r}} y_{1_{r}} y_{2_{r}}-k_{x} \dot{x}_{2_{r}}, \\
& \dot{u}_{y}=-\left(2 x_{1} y_{1_{r}}+\gamma_{1}\right)\left(\dot{x}_{2_{r}}+k_{y} x_{2_{r}}\right)-\left(3 y_{1_{r}}^{2}+x_{1_{r}}^{2}-\mu_{1}\right)\left(\dot{y}_{2_{r}}+k_{y} y_{2_{r}}\right)-2 y_{1_{r}}\left(x_{2_{r}}^{2}+3 y_{2_{r}}^{2}\right)-4 y_{2_{r}} x_{1_{r}} x_{2_{r}}-k_{y} \dot{y}_{2_{r}}, \\
& \dot{u}_{z}=-\left(\delta_{z}-g\right)\left(k+k_{z}\right)-k_{z} k z_{2_{r}},
\end{aligned}
$$

and $\dot{v}_{x}, \dot{v}_{y}, \dot{v}_{z}$ are defined as

$$
\begin{aligned}
& \dot{v}_{x}=-\left(3 x_{1_{r}}^{2}+y_{1_{r}}^{2}-\mu_{2}\right)\left(\dot{x}_{2_{r}}+\bar{k}_{x} x_{2_{r}}\right)-\left(2 x_{1, r} y_{1_{r}}-\gamma_{2}\right)\left(\dot{y}_{2_{r}}+\bar{k}_{x} y_{2_{r}}\right)-2 x_{1_{r}}\left(y_{2_{r}}^{2}+3 x_{2_{r}}^{2}\right)-4 x_{2_{r}} y_{1_{r}} y_{2_{r}}-\bar{k}_{x} \dot{x}_{2_{r}}, \\
& \dot{v}_{y}=-\left(2 x_{1_{r}} y_{1_{r}}+\gamma_{2}\right)\left(\dot{x}_{2 r}+\bar{k}_{y} x_{2_{r}}\right)-\left(3 y_{1_{r}}^{2}+x_{1_{r}}^{2}-\mu_{2}\right)\left(\dot{y}_{2_{r}}+\bar{k}_{y} y_{2_{r}}\right)-2 y_{1_{r}}\left(x_{2_{r}}^{2}+3 y_{2_{r}}^{2}\right)-4 y_{2_{r}} x_{1_{r}} x_{2_{r}}-k_{y} \dot{y}_{2_{r}}, \\
& \dot{v}_{z}=-\left(\delta_{z}-g\right)\left(\bar{k}+\bar{k}_{z}\right)-\bar{k}_{z} \bar{k} z_{2_{r}} .
\end{aligned}
$$




\section{Free initial trajectory conditions}

From the previous procedure, note that the initial conditions of the desired angles $\phi_{1_{r}}(0), \theta_{1_{r}}(0)$ and angular velocities $\phi_{2_{r}}(0), \theta_{2_{r}}(0)$ are clearly not always zero. For example at the first stage of movement (in numerical simulation), the initial angular position and velocity are given by $\phi_{1_{r}}(0)=59.7488^{\circ}, \theta_{1_{r}}(0)=30.6205^{\circ}, \phi_{2_{r}}(0)=-207.6294^{\circ} \mathrm{s}^{-1}$ and $\theta_{2 r}(0)=57.0956^{\circ} \mathrm{s}^{-1}$ respectively. Therefore in real-time experiments this could be a problem if a faster convergence for $\phi_{1} \rightarrow \phi_{1_{r}}$ and $\theta_{1} \rightarrow \theta_{1_{r}}$ is desired because it could compromise the system stability.

To solve this problem, four angular variables $\phi_{1_{f}}, \theta_{1_{f}}, \dot{\phi}_{1_{f}}=\phi_{2_{f}}$ and $\dot{\theta}_{1_{f}}=\theta_{2_{f}}$ are used, such that the initial conditions can be chosen as $\phi_{1_{f}}(0)=0^{\circ}, \theta_{1_{f}}(0)=0^{\circ}$ and $\phi_{2_{f}}(0)=0^{\circ} \mathrm{s}^{-1}, \theta_{2_{f}}(0)=0^{\circ} \mathrm{s}^{-1}$.

These variables are defined as:

$\dot{\phi}_{1_{f}}=\phi_{2_{f}}$

$\dot{\phi}_{2_{f}}=\left[\dot{\phi}_{2 r}-c_{1}\left(\phi_{2_{f}}-\phi_{2_{r}}\right)-c_{1_{f}} s_{\phi_{1}}\right] \tilde{g}(t)+\left[\dot{\phi}_{2_{r}}-c_{2}\left(\phi_{2_{f}}-\phi_{2_{r}}\right)-c_{2_{f}} \sigma_{\phi_{1 f}}\right] \tilde{f}(t) \bar{g}(t)+\left[\dot{\phi}_{2 r}-c_{3}\left(\phi_{2_{f}}-\phi_{2_{r}}\right)-c_{3_{f}} \Sigma_{\phi_{1 f}}\right] \bar{f}(t)$

$\dot{\theta}_{1_{f}}=\theta_{2_{f}}$

$\dot{\theta}_{2_{f}}=\left[\dot{\theta}_{2_{r}}-d_{1}\left(\theta_{2_{f}}-\theta_{2_{r}}\right)-d_{1_{f}} s_{\theta_{1}}\right] \tilde{g}(t)+\left[\dot{\phi}_{2_{r}}-d_{2}\left(\theta_{2_{f}}-\theta_{2_{r}}\right)-d_{2_{f}} \sigma_{\theta_{1}}\right] \tilde{f}(t) \bar{g}(t)+\left[\dot{\phi}_{2 r}-d_{3}\left(\theta_{2_{f}}-\theta_{2_{r}}\right)-d_{3_{f}} \Sigma_{\theta_{1_{f}}}\right] \bar{f}(t)$

with

$$
\begin{array}{lll}
S_{\phi_{1_{f}}}=\left(\phi_{2_{f}}-\phi_{2_{r}}\right)+c_{1}\left(\phi_{1_{f}}-\phi_{1_{r}}\right), & \sigma_{\phi_{1_{f}}}=\left(\phi_{2_{f}}-\phi_{2_{r}}\right)+c_{2}\left(\phi_{1_{f}}-\phi_{1_{r}}\right), & \Sigma_{\phi_{1_{f}}}=\left(\phi_{2_{f}}-\phi_{2_{r}}\right)+c_{3}\left(\phi_{1_{f}}-\phi_{1_{r}}\right), \\
S_{\theta_{1_{f}}}=\left(\theta_{2_{f}}-\theta_{2_{r}}\right)+d_{1}\left(\theta_{1_{f}}-\theta_{1_{r}}\right), & \sigma_{\theta_{1_{f}}}=\left(\theta_{2_{f}}-\theta_{2_{r}}\right)+d_{2}\left(\theta_{1_{f}}-\theta_{1_{r}}\right), & \Sigma_{\theta_{1_{f}}}=\left(\theta_{2_{f}}-\theta_{2_{r}}\right)+d_{3}\left(\theta_{1_{f}}-\theta_{1_{r}}\right),
\end{array}
$$

where $c_{i}, d_{i}$, and $c_{i_{f}}, d_{i_{f}}, i: 1,2,3$ are constant gains.

From the previous notice that $\phi_{1_{f}} \rightarrow \phi_{1_{r}}, \theta_{1_{f}} \rightarrow \theta_{1_{r}}$ and $\phi_{2_{f}} \rightarrow \phi_{2_{r}}, \theta_{2_{f}} \rightarrow \theta_{2_{r}}$ asymptotically.

One advantage when using these variables for tracking is that, for each time interval, asymptotic convergence can be achieved as fast as desired, and it will only depend on the gains chosen for each time interval. For example when $T_{F 1}=T_{F 2}$, (corresponding to the first scenario) it is convenient to choose small gains $c_{1}, c_{1_{f}}$. In contrast, $d_{1}, d_{1_{f}}$ are preferred for the time interval $0 \leq t<T_{F 1}$, due to the high initial angular conditions. For all $T_{F 1} \leq t<T_{F}$ is favorable to choose high gains $c_{2}, c_{2_{f}}$ and $d_{2}, d_{2_{f}}$, since in this time interval the quadrotor motion has been switched from take-off to a desired altitude $h$ to a landing spiral motion around $\left(0,0, z_{1_{r}}(0)\right)$. This means that it is convenient to propose high gains when the dynamics are being switched.

The next step is to propose an attitude controller such that, $\phi_{1} \rightarrow \phi_{1_{f}}, \theta_{1} \rightarrow \theta_{1_{f}}$ asymptotically, and in consequence it will follow that $\phi_{1} \rightarrow \phi_{1_{r}}, \theta_{1} \rightarrow \theta_{1_{r}}$ asymptotically.

\section{Attitude nonlinear controller}

Observe from (4)-(6) that the attitude dynamics are represented by a nonlinear MIMO system.

Define now $e_{\phi_{1}}=\phi_{1}-\phi_{1_{f}} ; e_{\theta_{1}}=\theta_{1}-\theta_{1_{f}} ; e_{\psi_{1}}=\psi_{1}-\psi_{1_{r}}$, thefore $e_{\phi_{2}}=\phi_{2}-\phi_{2_{f}} ; e_{\theta_{2}}=\theta_{2}-\theta_{2_{f}}$ and $e_{\psi_{2}}=\psi_{2}-\dot{\psi}_{1_{r}}$. The desired yaw angle $\psi_{1 r}$ is considered constant such that $\dot{\psi}_{1_{r}}=\ddot{\psi}_{1 r}=0$. Hence the following system can be stated:

$$
\begin{aligned}
& \dot{e}_{\phi_{1}}=e_{\phi_{2}} \quad, \quad \dot{e}_{\theta_{1}}=e_{\theta_{2}} \quad, \quad \dot{e}_{\psi_{1}}=e_{\psi_{2}}, \\
& \dot{e}_{\phi_{2}}=\bar{f}_{1}+b_{1} U_{\phi}+\xi_{1} \quad, \quad \dot{e}_{\theta_{2}}=\bar{f}_{2}+b_{2} U_{\theta}+\xi_{2} \quad, \quad \dot{e}_{\psi_{2}}=\bar{f}_{3}+b_{3} U_{\psi}+\xi_{3},
\end{aligned}
$$


with $\bar{f}_{1}=\theta_{2}\left(\psi_{2} \varpi_{1}-\beta_{1}\right)-\dot{\phi}_{2_{f}}, \bar{f}_{2}=\phi_{2}\left(\psi_{2} \varpi_{2}-\beta_{1}\right)-\dot{\theta}_{2 f}, \bar{f}_{3}=\theta_{2} \phi_{2} \varpi_{3}$. And $\varpi_{1}=\left(\frac{I_{y}-I_{X}}{I_{x}}\right), \varpi_{2}=\left(\frac{I_{z}-I_{x}}{I_{y}}\right)$, $\varpi_{3}=\left(\frac{I_{x}-I_{y}}{I_{z}}\right), \beta_{1}=\frac{I_{r}}{I_{x}} \Omega, \beta_{2}=\frac{I_{r}}{I_{y}} \Omega, b_{1}=\frac{l}{I_{x}}, b_{2}=\frac{l}{I_{y}}, b_{3}=\frac{l}{I_{z}}$.

Notice that (27) can also be written as

$$
\dot{\boldsymbol{x}}=A \boldsymbol{x}+B(\boldsymbol{u}+\overline{\boldsymbol{\xi}})=\left(\begin{array}{cc}
0_{3 \times 3} & \mathbb{\Xi}_{3 \times 3} \\
0_{3 \times 3} & 0_{3 \times 3}
\end{array}\right)\left(\begin{array}{c}
\boldsymbol{q} \\
\boldsymbol{v}_{\boldsymbol{q}}
\end{array}\right)+\left(\begin{array}{c}
0_{3 \times 3} \\
B_{2}
\end{array}\right)(\boldsymbol{u}+\overline{\boldsymbol{\xi}})
$$

with $\boldsymbol{q}=\left(e_{\phi_{1}}, e_{\theta_{1}}, e_{\psi_{1}}\right)^{T}, \boldsymbol{v}_{\boldsymbol{q}}=\left(e_{\phi_{2}}, e_{\theta_{2}}, e_{\psi_{2}}\right)^{T}, \boldsymbol{u}=\left(U_{\phi}, U_{\theta}, U_{\psi}\right)^{T}, \boldsymbol{x}=\left(\boldsymbol{q}, \boldsymbol{v}_{\boldsymbol{q}}\right)^{T}$ and

$$
B_{2}=\left(\begin{array}{ccc}
b_{1} & 0 & 0 \\
0 & b_{2} & 0 \\
0 & 0 & b_{3}
\end{array}\right), \quad A=\left(\begin{array}{cc}
0_{3 \times 3} & \mathbb{1}_{3 \times 3} \\
0_{3 \times 3} & 0_{3 \times 3}
\end{array}\right), \quad \bar{\xi}=\left(\frac{\bar{f}_{1}+\xi_{1}}{b_{1}}, \frac{\bar{f}_{2}+\xi_{2}}{b_{2}}, \frac{\bar{f}_{3}+\xi_{3}}{b_{3}}\right)^{T}
$$

Remark that (27) is now expressed as a perturbed linear MIMO system by (28) without linearization around equilibrium points.

\section{Sliding mode surfaces design}

Considering that the quadrotor has a balanced configuration such that its inertial effects mainly affect its symmetrical axes, thus propose

$$
\boldsymbol{S}=\boldsymbol{v}_{\boldsymbol{q}}+M \boldsymbol{q}=\boldsymbol{v}_{\boldsymbol{q}}+\left(\begin{array}{ccc}
M_{11} & 0 & 0 \\
0 & M_{22} & 0 \\
0 & 0 & M_{33}
\end{array}\right) \boldsymbol{q}
$$

where $M$ is a diagonal matrix with positive values $M_{11}, M_{22}, M_{33}$. Then $S_{i}=e_{j_{i+1}}+M_{i i} e_{j_{i}}$ for $i=1,2,3$ and $j: \phi, \theta, \psi$.

Observe that when $U_{j}$ achieves $S_{i} \rightarrow 0$ in finite time $t_{r_{i}}$ then the sliding modes appear, and these are given by

$$
\begin{array}{lll}
e_{\phi_{1}}(t)=e^{-M_{11}\left(t-t_{r_{1}}\right)} e_{\phi_{1}}\left(t_{r_{1}}\right), & e_{\theta_{1}}(t)=e^{-M_{22}\left(t-t_{r_{2}}\right)} e_{\theta_{1}}\left(t_{r_{2}}\right), & e_{\psi_{1}}(t)=e^{-M_{33}\left(t-t_{r_{3}}\right)} e_{\psi_{1}}\left(t_{r_{3}}\right), \\
e_{\phi_{2}}(t)=-M_{11} e^{-M_{11}\left(t-t_{r_{1}}\right)} e_{\phi_{1}}\left(t_{r_{1}}\right), & e_{\theta_{2}}(t)=-M_{22} e^{-M_{22}\left(t-t_{r_{2}}\right)} e_{\theta_{1}}\left(t_{r_{2}}\right), & e_{\psi_{2}}(t)=-M_{33} e^{-M_{33}\left(t-t_{r_{3}}\right)} e_{\psi_{1}}\left(t_{r_{3}}\right) .
\end{array}
$$

Taking the time derivative of $S$

$$
\dot{\boldsymbol{S}}=B_{2}(\boldsymbol{u}+\overline{\boldsymbol{\xi}})+M \boldsymbol{v}_{\boldsymbol{q}}
$$

then, introducing $\boldsymbol{u}=B_{2}^{-1}\left\{\overline{\boldsymbol{u}}-\boldsymbol{M} \boldsymbol{v}_{q}\right\}$ for removing the linear part, it implies that

$$
\dot{\boldsymbol{S}}=\overline{\boldsymbol{u}}+B_{2} \overline{\boldsymbol{\xi}},
$$

or in the scalar form

$$
\dot{S}_{i}=\bar{u}_{i}+\bar{f}_{i}+\xi_{i}, \quad i=1,2,3 .
$$




\section{| Integral sliding mode controller}

Notice from (34), that controller $\bar{u}_{i}$ must be proposed to compensate, on one hand, nonlinearities $\bar{f}_{i}$ and unknown and external perturbations $\xi_{i}$ from the beginning, and on the other hand, to ensure that each sliding surfaces goes to zero.

Then, it is possible to consider a controller composed by two parts as

$$
\bar{u}_{i}=\bar{u}_{i_{1}}+\bar{u}_{i_{2}} .
$$

Proposing a sliding surface as $\sigma_{i}=S_{i}-Z_{i}$ with $\dot{Z}_{i}=\bar{u}_{i_{2}}$, the following candidate Lyapunov function can be introduced

$$
V\left(\sigma_{i}\right)=\frac{1}{2} \sigma_{i}^{2}>0
$$

For proving global asymptotic stability for $\sigma_{i}$ at the equilibrium point $\sigma_{i}=0$, the following conditions must be satisfied a) $\lim _{\left|\sigma_{i}\right| \rightarrow \infty} V=\infty$, and $\left.b\right) \dot{V}<0$ for $\sigma_{i} \neq 0$.

Condition (a) is obviously satisfied by $V$ in (36). In order to achieve finite-time convergence (global finite-time stability), condition (b) can be modified as

$$
\dot{V} \leq-\alpha_{i} V^{1 / 2}, \quad \alpha_{i}>0
$$

Indeed, separating variables and integrating inequality (37) over time interval $0 \leq \tau \leq t$ yields

$$
V^{1 / 2}\left(\sigma_{i}(t)\right)-V^{1 / 2}\left(\sigma_{i}(0)\right) \leq-\frac{1}{2} \alpha_{i} t
$$

and considering that $V\left(\sigma_{i}(t)\right)$ reaches zero in finite time $t_{r}$, it follows that $V^{1 / 2}\left(\sigma_{i}\left(t_{r}\right)\right)-V^{1 / 2}\left(\sigma_{i}(0)\right) \leq-\frac{1}{2} \alpha_{i} t_{r}$. This implies that

$$
t_{r} \leq \frac{2 V^{1 / 2}\left(\sigma_{i}(0)\right)}{\alpha_{i}}
$$

Notice now that (37) can be written as

$$
\sigma_{i} \dot{\sigma}_{i} \leq-\bar{\alpha}_{i}\left|\sigma_{i}\right|, \quad \bar{\alpha}_{i}=\frac{\alpha_{i}}{\sqrt{2}}>0
$$

From (34) and (35), it follows that

$$
\sigma_{i} \dot{\sigma}_{i}=\sigma_{i}\left(\dot{S}_{i}-\dot{Z}_{i}\right)=\sigma_{i}\left(\bar{u}_{i_{1}}+\bar{f}_{i}+\xi_{i}\right)
$$

Real-world physical systems are usually constrained by inputs or states that cannot be neglected. These can be introduced by actuator or sensor range limitations. Some constraints are inherent limits (for example actuators in electromechanical systems) that depend on the system configuration and cannot be physically violated.

This implies that such systems cannot be endured by infinite perturbations. For the presented case of study, the external perturbations are unknown, but it is possible to consider a bound given by the system's physical limitations. Therefore for control purposes each $\xi_{i}$ is considered to be bounded, i.e. $\left|\xi_{i}\right| \leq L_{i}$, where $L_{i}$ defines the bound that the 
system could hold by its physical configuration. The disturbances bounds can be selected using several approaches, for many implementations, an empirical selection of such values would guarantee the compensation of most disturbances, alternatively, adaptive techniques using terminal sliding modes can be applied to autonomously identify such parameters, as proposed by [33] and [34].

Selecting $\bar{u}_{i_{1}}=-\rho_{i} \operatorname{sign}\left(\sigma_{i}\right)$, and using (41), condition (40) is fulfilled if and only if

$$
\rho_{i}=\bar{\alpha}_{i}+\left|\bar{f}_{i}\right|+L_{i}
$$

where (42) represents the necessary gains for ensuring stability in a bounded finite time $t_{r}$, meaning that

$$
t_{r} \leq \frac{2 V^{1 / 2}\left(\sigma_{i}(0)\right)}{\alpha_{i}}=\frac{\sqrt{2}\left|\sigma_{i}(0)\right|}{\bar{\alpha}_{i}} .
$$

The above implies that $\sigma_{i}=\dot{\sigma}_{i}=0$ for all $t \geq t_{r}$, hence condition $\dot{\sigma}_{i}=0$ produces

$$
\dot{\sigma}_{i}=\underbrace{-\rho_{i} \operatorname{sign}\left(\sigma_{i}\right)}_{\bar{u}_{i_{1}}}+\bar{f}_{i}+\xi_{i}=0 \quad \forall t \geq t_{r} .
$$

This means that $-\rho_{i} \operatorname{sign}\left(\sigma_{i}\right)$ compensates the disturbance terms $\bar{f}_{i}+\xi_{i}$ only during the reaching phase.

Observe from (43) that proposing $\sigma_{i}(0)=0$ implies that $t_{r}=0$, such that $\sigma_{i}=\dot{\sigma}_{i}=0$ for all $t \geq 0$. Thus

$$
\bar{u}_{i_{1}}=-\rho_{i} \operatorname{sign}\left(\sigma_{i}\right)=-\left(\bar{f}_{i}+\xi_{i}\right), \quad \forall t \geq 0,
$$

therefore controller $\bar{u}_{i_{1}}$ compensates $\bar{f}_{i}+\xi_{i}$ for all $t \geq 0$ if and only if $\sigma_{i}(0)=0$.

Considering now that $\bar{u}_{i 1}$ accomplishes $\sigma_{i}(t)=0, \forall t \geq 0$, then it follows that $S_{i}(t)=Z_{i}(t)$ for all $t \geq 0$, consequently the sliding surface can be rewritten as

$$
\begin{aligned}
& S_{i}=Z_{i} \\
& \dot{Z}_{i}=\bar{u}_{i_{2}} \text { with } \quad Z_{i}(0)=S_{i}(0) .
\end{aligned}
$$

From the above, it yields that $\dot{S}_{i}=\bar{u}_{i_{2}}$. Proposing $\bar{u}_{i_{2}}=-k_{i}\left|S_{i}\right|^{1 / 2} \operatorname{sign}\left(S_{i}\right)$, a Lyapunov candidate function can be defined to prove convergence of (44) to zero as

$$
V\left(S_{i}\right)=\left|S_{i}\right|>0,
$$

once again, the following conditions must be satisfied a) $\lim _{\left|S_{i}\right| \rightarrow \infty} V=\infty$ and $\left.b\right) \dot{V}<0$ for $S_{i} \neq 0$.

Condition (a) is satisfied according to (45), now for proving global finite-time stability at $S_{i}=0$, a modified condition will be used as

$$
\dot{V} \leq-\alpha_{i} V^{1 / 2}, \quad \alpha_{i}>0
$$

introducing (45) into (46), an equivalent modified condition is obtained as

$$
\frac{S_{i} \dot{S}_{i}}{\left|S_{i}\right|} \leq-\alpha_{i}\left|S_{i}\right|^{1 / 2} .
$$


Since $\dot{S}_{i}=\bar{u}_{i_{2}}$, then (47) finally yields

$$
-k_{i}\left|S_{i}\right|^{1 / 2} \leq-\alpha_{i}\left|S_{i}\right|^{1 / 2}
$$

implying that each gain $k_{i}$ must be equal to $\alpha_{i}$, such that

$$
\dot{V}=-\alpha_{i} V^{1 / 2}, \text { if } k_{i}=\alpha_{i}>0 .
$$

Separating variables and integrating inequality (49) over the time interval $0 \leq \tau \leq t$, it yields

$$
V^{1 / 2}\left(S_{i}(t)\right)-V^{1 / 2}\left(S_{i}(0)\right)=-\frac{1}{2} \alpha_{i} t
$$

observe that $V\left(S_{i}(t)\right)$ reaches zero at time $t_{r_{i}}$, therefore it follows that

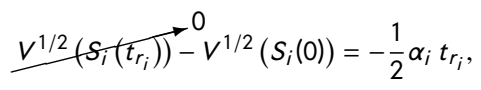

which implies that

$$
t_{r_{i}}=\frac{2 V^{1 / 2}\left(s_{i}(0)\right)}{k_{i}}=\frac{2\left|s_{i}(0)\right|^{1 / 2}}{k_{i}},
$$

Consequently $S_{i}(t) \rightarrow 0$ in finite time $t_{r_{i}}$ and $\bar{u}_{i_{2}}$ keeps them at zero thereafter.

The previous means that $S_{i}=0 \forall t \geq 0$, signifying that robustness and sliding modes convergence are reached since the beginning. The previous also implies that $e_{\phi}, e_{\theta}$ and $e_{\psi}$ go to zero and $\phi \rightarrow \phi_{1_{f}}, \theta \rightarrow \theta_{1_{f}}$ and $\psi \rightarrow \psi_{1_{f}}$ and thus, $\phi \rightarrow \phi_{r}, \theta \rightarrow \theta_{1}$ and $\psi \rightarrow \psi_{1}$, holding Assumption 1 .

An advantage when designing $\phi_{1_{f}}, \theta_{1_{f}}, \phi_{2_{f}}, \theta_{2_{f}}$ altogether with the integral sliding mode control is that the occurence of the sliding modes can be achieved since the beginning, guaranteeing angular stabilization also from the start.

Remark From the fact that $S_{i}=0$ for all $t \geq 0$ then, the coefficients $k_{i}$ in $\bar{u}_{i_{2}}$ can be proposed with small values in order to diminish the scope of the controllers without compromising stability.

\section{NUMERICAL VALIDATION}

The dynamic trajectory and the control algorithm were validated in numerical simulations, the constant parameters and gains were selected empirically such that the simulation performance resembled the behavior of real world quadrotors, such values are depicted in Table 2, while the system initial conditions were set as detailed in Table 3 .

\begin{tabular}{|l|c|c|c|c|c|c|c|}
\hline$x_{1_{r}}(0)=0.23 m$ & $x_{2 r}(0)=0 \mathrm{~m} / \mathrm{s}$ & $n=2$ & $\mu_{1}=4$ & $\mu_{2}=-0.20$ & $k_{x}=1$ & $\bar{k}_{x}=12$ & $T_{F}=50 s$ \\
\hline$y_{1_{r}}(0)=-1.0 m$ & $y_{2 r}(0)=0 \mathrm{~m} / \mathrm{s}$ & $T_{F 1}=15 \mathrm{~s}$ & $\gamma_{1}=-1$ & $\gamma_{2}=1$ & $k_{y}=1$ & $\bar{k}_{y}=12$ & $h=4 m$ \\
\hline$z_{1_{r}}(0)=0 \mathrm{~m}$ & $z_{2 r}(0)=0 \mathrm{~m} / \mathrm{s}$ & $T_{F 2}=35 \mathrm{~s}$ & $k=1$ & $\bar{k}=0.5$ & $k_{z}=4$ & $\bar{k}_{z}=0.5$ & \\
\cline { 1 - 5 } & &
\end{tabular}

TAB LE 2 Constant parameters and gains used for numerical simulations. 


\begin{tabular}{|c|c|c|c|}
\hline$x_{1}(0)=x_{1_{r}}(0)$ & $\phi_{1}(0)=\phi_{1_{f}}(0)=0$ & $x_{2}(0)=x_{2_{r}}(0)$ & $\phi_{2}(0)=\phi_{2_{f}}(0)=0$ \\
\hline$y_{1}(0)=y_{1_{r}}(0)$ & $\theta_{1}(0)=\theta_{1_{f}}(0)=0$ & $y_{2}(0)=y_{2_{r}}(0)$ & $\theta_{2}(0)=\theta_{2_{f}}(0)=0$ \\
\hline$z_{1}(0)=z_{1_{r}}(0)$ & $\psi_{1}(0)=0$ & $z_{2}(0)=z_{2_{r}}(0)$ & $\psi_{2}(0)=0$ \\
\hline
\end{tabular}

TABLE 3 Quadrotor initial conditions

The gains used for designing $\phi_{1_{f}}, \theta_{1_{f}}$ and $\phi_{2_{f}}, \theta_{2_{f}}$ are given in the Table 4 .

\begin{tabular}{|l|l|l|}
\hline $0 \leq t<T_{F 1}$ & $T_{F 1} \leq t<T_{F 2}$ & $T_{F 2} \leq t \leq T_{F}$ \\
\hline$c_{1}=c_{1_{f}}=4$ & $c_{2}=c_{2_{f}}=150$ & $c_{3}=c_{3_{f}}=10$ \\
\hline$d_{1}=d_{1_{f}}=4$ & $d_{2}=d_{2_{f}}=150$ & $d_{3}=d_{3_{f}}=10$ \\
\hline
\end{tabular}

TAB LE 4 Gains used for different interval times in the trajectory design.

The gains for the sliding surfaces are selected as $M_{11}=10, M_{22}=10, M_{33}=4, k_{1}=k_{2}=k_{3}=0.45$, and $\alpha_{1}=0.1$, $\alpha_{2}=0.1, \alpha_{3}=0.1$.

For simulation purposes, the external and unknown perturbations were considered as

$$
\begin{aligned}
& \xi_{1}=2 \sin (t) \operatorname{sign}\left(S_{1}\right) \\
& \xi_{2}=-1.5 \cos (2 t) \operatorname{sign}\left(S_{2}\right) \\
& \xi_{3}=-0.5 e^{\cos (t)} \operatorname{sign}\left(S_{3}\right)
\end{aligned} \quad\left\{\begin{array}{l}
\left|\xi_{1}\right| \leqq L_{1}=2 \\
\left|\xi_{2}\right| \leqq L_{2}=1.5 \\
\left|\xi_{3}\right| \leqq L_{3}=0.5 e^{1}
\end{array}\right.
$$

The performance of these perturbations are depicted in Figure 8. Notice that they present an evident chattering effect caused by the term $\operatorname{sign}\left(S_{i}\right)$, beginning at the convergence time $t_{r_{i}}=0$. Nevertheless observe also that, although these uncertainties present a high frequency the control signals are able of compensating such perturbations since the initial time instant $t=0$.
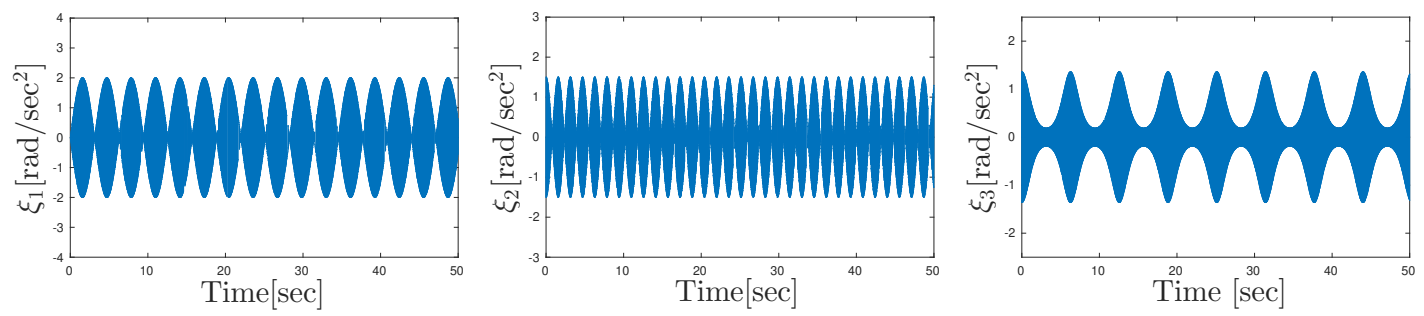

FIGURE 8 Uncertainties performance $\xi_{1}, \xi_{2}$ and $\xi_{3}$.

The following graphs were obtained when simulating the proposed control-scheme. In Figure 9 the dynamic trajectory (red dotted line) and the quadrotor performance (blue solid line) are introduced. On one hand, observe the good performance of the nonlinear controller when following the desired trajectory even in presence of unknown and external disturbances, in the other hand, notice that the trajectory is well defined and planned. In Figure 10 the translational errors (position and velocity) are depicted, observe that these behaviors are small and with an asymptotic 


\section{convergence to zero.}

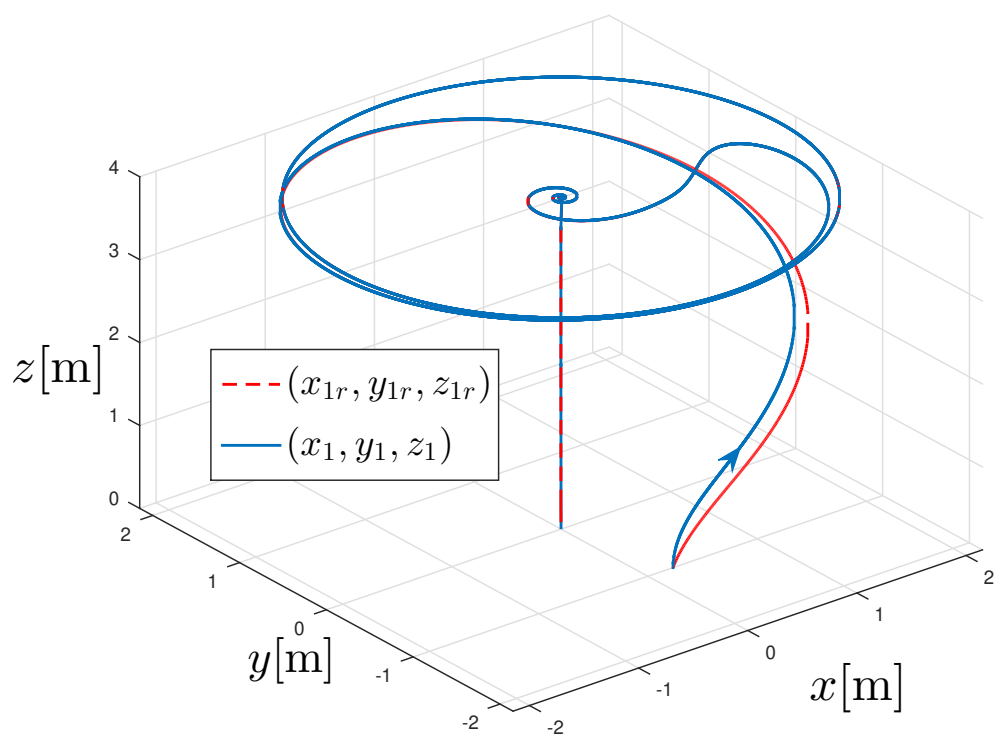

FIGURE 9 3D quadrotor performance when tracking the proposed trajectory. 

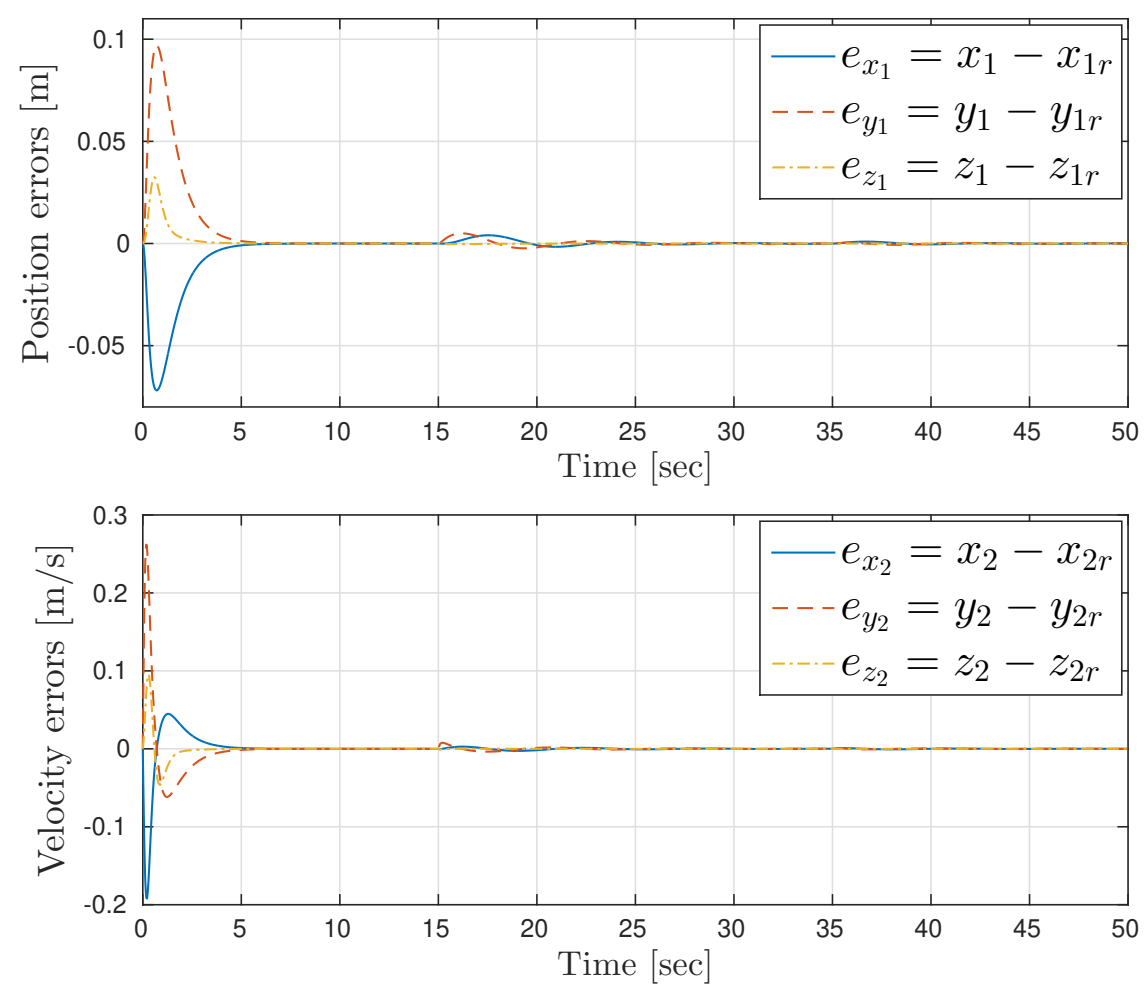

FIGURE 10 Translational errors performances when the quadrotor tracks the proposed trajectory.

Sliding surfaces $\sigma_{1}, \sigma_{2}, \sigma_{3}$ and $S_{1}, S_{2}, S_{3}$ become zero since the initial time, this means that $\sigma_{i}=0 ; \forall t \geq 0$ guarantees robustness respect to uncertainties from start to finish and $S_{i}=0 ; \forall t \geq 0$ guarantees the asymptotic stabilization of $e_{\phi_{1}}, e_{\theta_{1}}, e_{\psi_{1}}$ and its velocities from the beginning as can be seen in Figure 11. Notice here that the errors remain close to zero. 

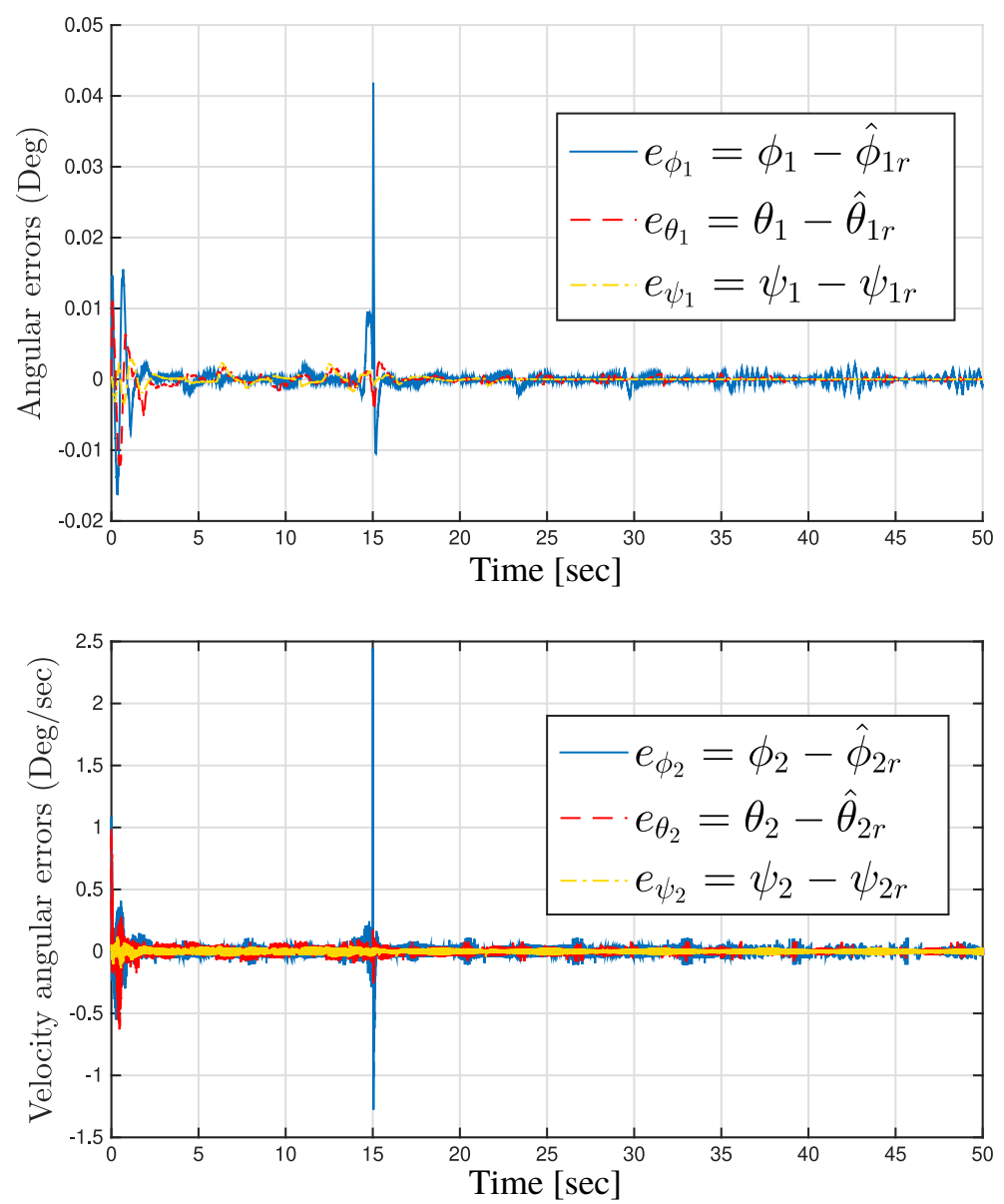

FIGURE 11 Angular errors performance when applying the proposed control strategy. Note that a switch from circular surveillance to centering stage happens at $T_{F 1}=15 \mathrm{~s}$, which induces a small peak in the angular errors.

In Figure 12 the asymptotic convergence of $\phi_{1_{f}} \rightarrow \phi_{1_{r}}$ and $\theta_{1_{f}} \rightarrow \theta_{1_{r}}$ is appreciated by using (25) and (26), in addition remember that $U_{\phi}, U_{\theta}$ are necessary to achieve $e_{\phi_{1}}=\phi_{1}-\phi_{1_{f}} \rightarrow 0$ and $e_{\theta_{1}}=\theta_{1}-\theta_{1_{f}} \rightarrow 0$ asymptotically, implying also $\phi_{1} \rightarrow \phi_{1}$ and $\theta_{1} \rightarrow \theta_{1}$ asymptotically, therefore Assumption 4.1 is held.

In the following figures, the control behavior is depicted, notice in Figure 13 that these control signals present a chattering effect from the beginning, this means that the algorithms are compensating the proposed unknown uncertainties $\xi_{1}, \xi_{2}$ and $\xi_{3}$ for all $t \geq 0$. Similarly, in Figure 14 it is possible to analyze the good performance of the position control $U_{1}$ while achieving trajectory tracking. 

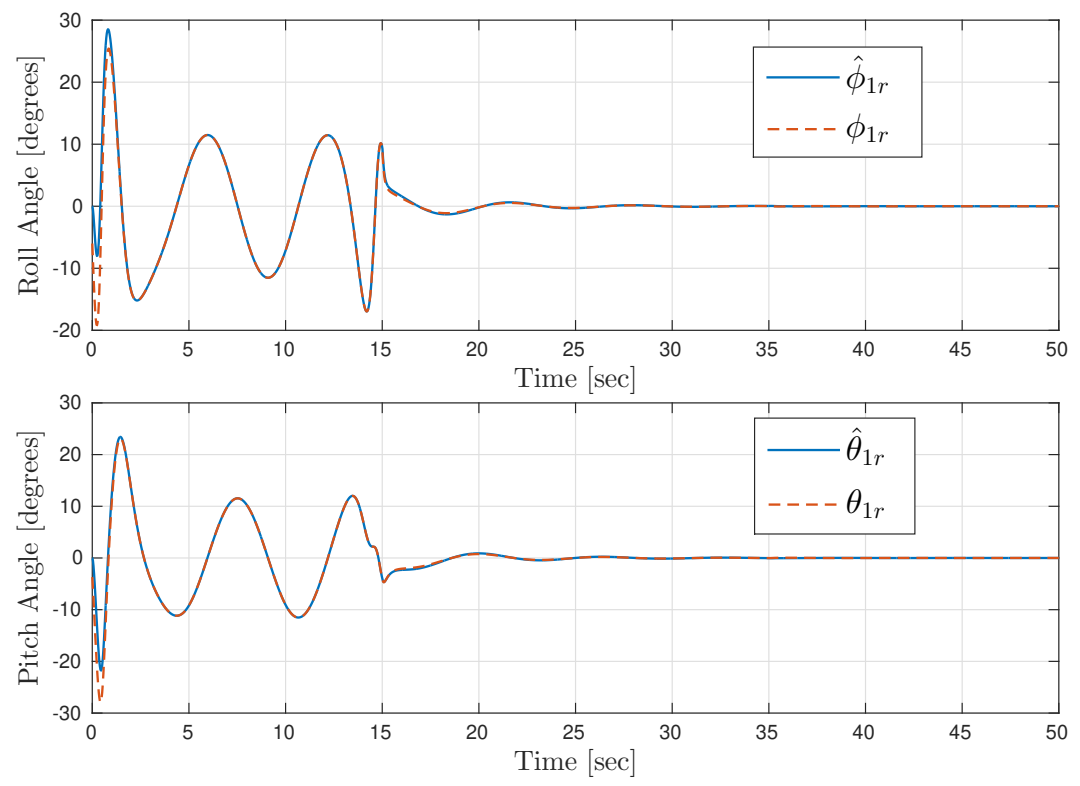

FIGURE 12 Fast converge of the virtual angular variables to their references, i.e. $\phi_{1_{f}} \rightarrow \phi_{1_{r}}$ and $\theta_{1_{f}} \rightarrow \theta_{1_{r}}$
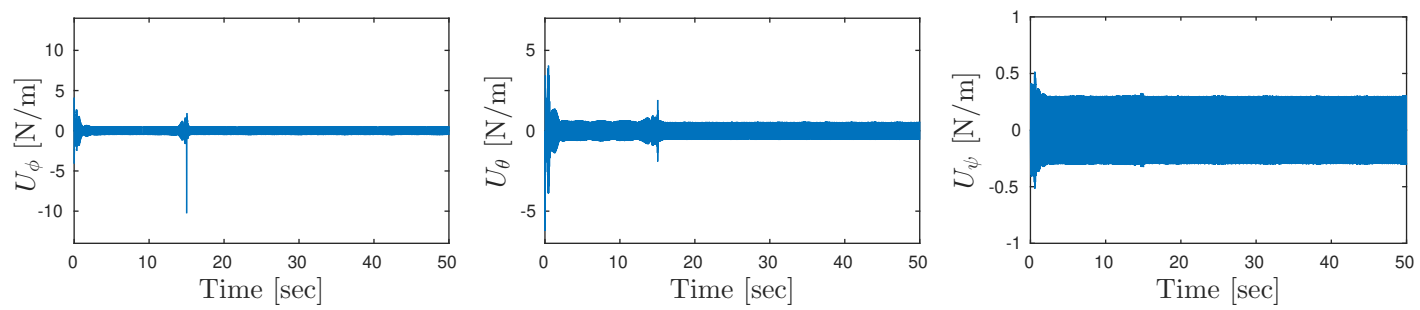

FIGURE 13 Attitude control signals performance; $U_{\phi}, U_{\theta}$ and $U_{\psi}$.

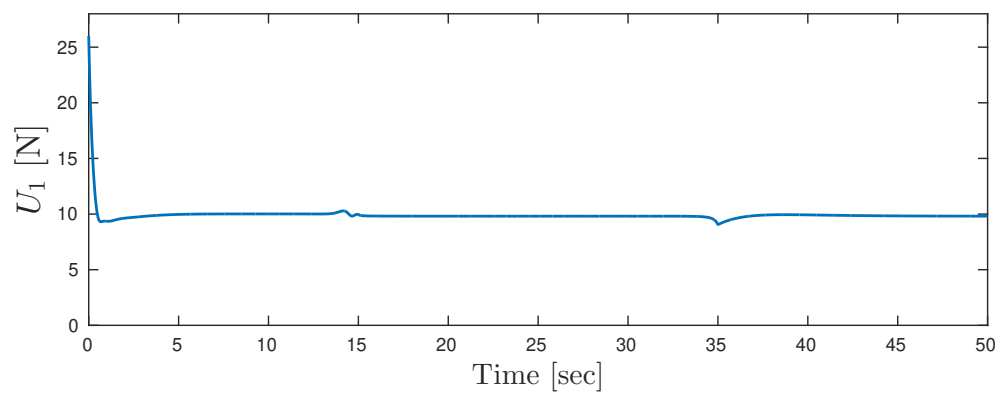

FIGURE 14 Position control behavior. 


\section{EXPERIMENTAL VALIDATION}

The controllers have also been validated in flight tests. The quadrotor used in this experiment was an AR Drone with an embedded ARM Cortex A8 processor at $1 \mathrm{GHz}$, running Linux 2.6.32, it also has a 1GB DDR2 RAM at $200 \mathrm{MHz}$, the sampling frequency is $200 \mathrm{~Hz}$. The employed navigation sensors were an Inertial Measurement Unit, as well as an OptiTrack Motion Capture system for position and yaw feedback. The hardware of the quadrotor has been conserved from its factory design, nevertheless the original software has been completely replaced by our framework FL-Air, such that custom programs can be run, accessing all states and variables that usually can not be modified with a brand new Parrot.

Notice that matrix $M$ was simplified (by considering only parameters in the main diagonal) for facilitating tuning, nevertheless, some nonlinear parameters are not easy to obtain because they depend on each prototype (form, configuration, material, etc) and they could change every time the prototype is modified. These parameters $\left(\beta_{2}, \varpi_{1}, \varpi_{2}, b_{1}\right)$ are represented by the following relations

$$
\begin{aligned}
& \rho_{1}=\left(\frac{\alpha_{1}}{\sqrt{2}}+\left|\left(e_{\theta_{2}}+\dot{\theta}_{1_{r}}\right)\left(e_{\psi_{2}} \varpi_{1}-\beta_{1}\right)-\ddot{\phi}_{1_{r}}\right|+L_{1}\right) \\
& \rho_{2}=\left(\frac{\alpha_{2}}{\sqrt{2}}+\left|\left(e_{\phi_{2}}+\dot{\phi}_{1_{r}}\right)\left(e_{\psi_{2}} \varpi_{2}-\beta_{2}\right)-\ddot{\theta}_{1_{r}}\right|+L_{2}\right) \\
& \rho_{3}=\left(\frac{\alpha_{3}}{\sqrt{2}}+\left|\left(e_{\theta_{2}}+\dot{\theta}_{1_{r}}\right)\left(e_{\phi_{2}}+\dot{\phi}_{1_{r}}\right) \varpi_{3}\right|+L_{3}\right)
\end{aligned}
$$

A procedure for easily tuning these kind of vehicles is proposed in the following. This idea will facilitate future practical validation when changing the prototype configuration. Rewriting the quadrotor parameters, it follows that

$$
\beta_{1}=\beta_{2}\left(\varpi_{1}+1\right), \quad b_{2}=\frac{b_{1}}{\left(\varpi_{1}+1\right)}, \quad b_{3}=\frac{b_{1}}{\left[\varpi_{2}\left(\varpi_{1}+1\right)+1\right]}, \quad \varpi_{3}=-\varpi_{1}\left(\frac{1}{\varpi_{2}\left(\varpi_{1}+1\right)+1}\right) .
$$

Hence, the goal will be to minimize the effect of nonlinear terms for the pitch $\left(e_{\theta_{2}}+\dot{\theta}_{1_{r}}\right)\left(e_{\psi_{2}} \varpi_{1}-\beta_{1}\right)$ and roll $\left(e_{\phi_{2}}+\dot{\phi}_{1_{r}}\right)\left(e_{\psi_{2}} \varpi_{2}-\beta_{2}\right)$ angles. Considering $\varpi_{1}, b_{1} \in \mathbb{R}$ can bee freely chosen, the following can be obtained:

$$
\varpi_{2}=\frac{\varpi_{1}}{\left(\varpi_{1}+1\right)}, \quad \varpi_{3}=-\frac{\varpi_{1}}{\varpi_{1}+1}, \quad b_{2}=b_{3}=\frac{b_{1}}{\left(\varpi_{1}+1\right)}
$$

this implies that

$$
\rho_{1}=\left(\frac{\alpha_{1}}{\sqrt{2}}+L_{1}+\left|\ddot{\phi}_{1_{r}}\right|\right), \quad \rho_{2}=\left(\frac{\alpha_{2}}{\sqrt{2}}+L_{2}+\left|\ddot{\theta}_{1_{r}}\right|\right), \quad \rho_{3}=\left(\frac{\alpha_{3}}{\sqrt{2}}+L_{3}+\left|\left(e_{\theta_{2}}+\dot{\theta}_{1_{r}}\right)\left(e_{\phi_{2}}+\dot{\phi}_{1_{r}}\right) \varpi_{3}\right|\right)
$$

Choosing $\varpi_{1}=0$ then $\varpi_{3}=0$ implies that

$$
\rho_{1}=\left(L_{x}+\left|\ddot{\phi}_{1 r}\right|\right), \quad \rho_{2}=\left(L_{y}+\left|\ddot{\theta}_{1_{r}}\right|\right), \quad \rho_{3}=\left(L_{z}\right),
$$

where $L_{x}=\frac{\alpha_{1}}{\sqrt{2}}+L_{1}, L_{y}=\frac{\alpha_{2}}{\sqrt{2}}+L_{2}$ and $L_{z}=\frac{\alpha_{3}}{\sqrt{2}}+L_{3}$. Therefore, the only parameters that must be experimentally tuned are $b_{i}, L_{i}, M_{i i}, K_{i}, i=1,2,3$, which then yield the other terms by computing the previous relations.

The flight tests consisted on taking-off until a desired altitude of $1.4 \mathrm{~m}$ is reached, then following a circular trajectory of $1 \mathrm{~m}$ of radius and finally landing, all in autonomous mode. The good performance of the control algorithm tracking the well defined dynamic trajectory is depicted in the following graphs. 
Figure 15 illustrates the quadrotor position response in $3 \mathrm{D}$ when during the flight tests. Notice also in Figure $16 \mathrm{a}$ $2 \mathrm{D}$ view from Figure 15, remark here the good real-time performance of the control scheme.

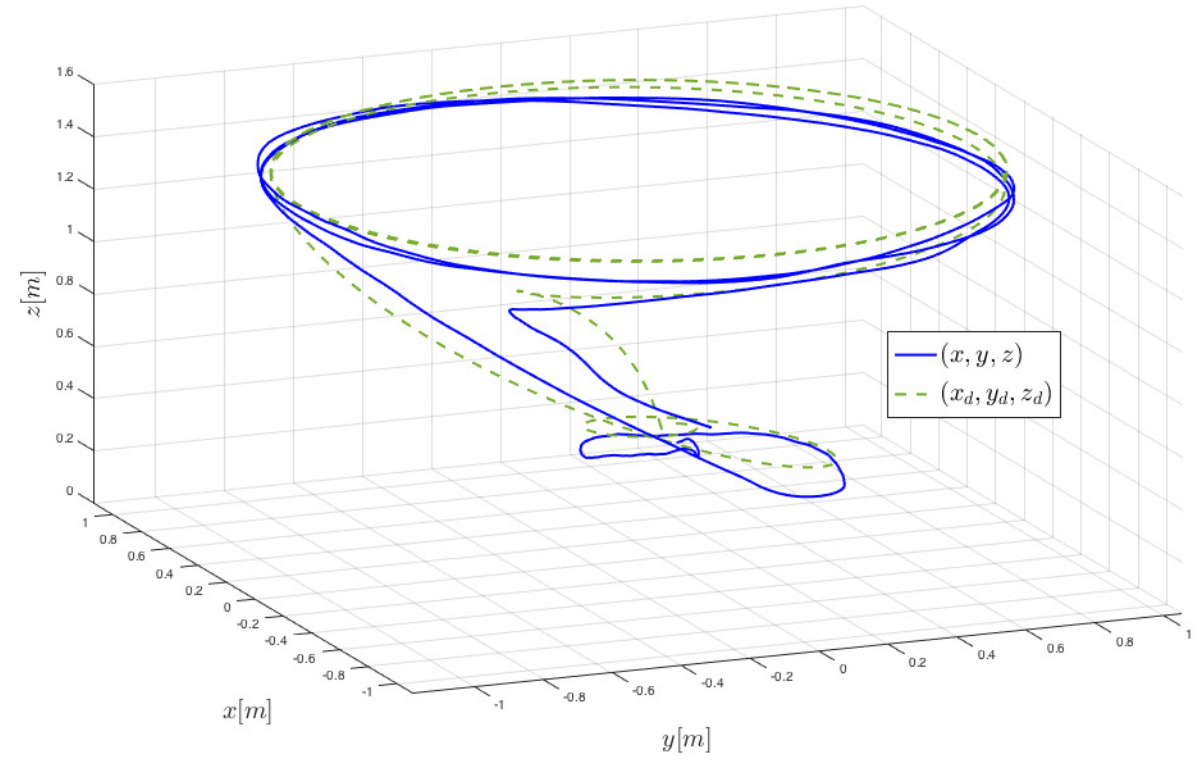

F IGURE 15 3D system response when tracking the proposed trajectory.

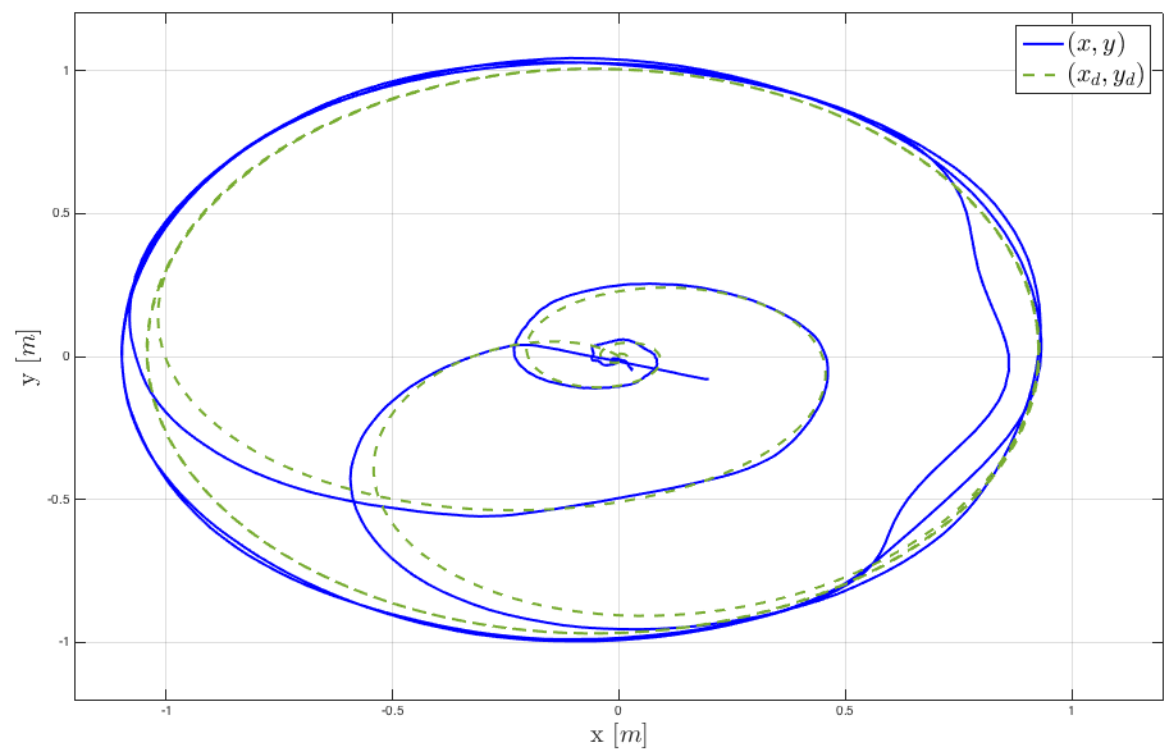

FIGURE 16 Quadrotor performance in the horizontal plane. 
From Figure 17 observe the quadrotor states behavior when following the references from the dynamic trajectory. Notice here the good performance of the controller for tracking $x_{1_{r}}, y_{1_{r}}$ and $z_{1_{r}}$. Similarly in Figure 18 the good performance of the controller can be analyzed when following the desired angles $\phi_{r}, \theta_{r}$ obtained from the dynamic trajectory.
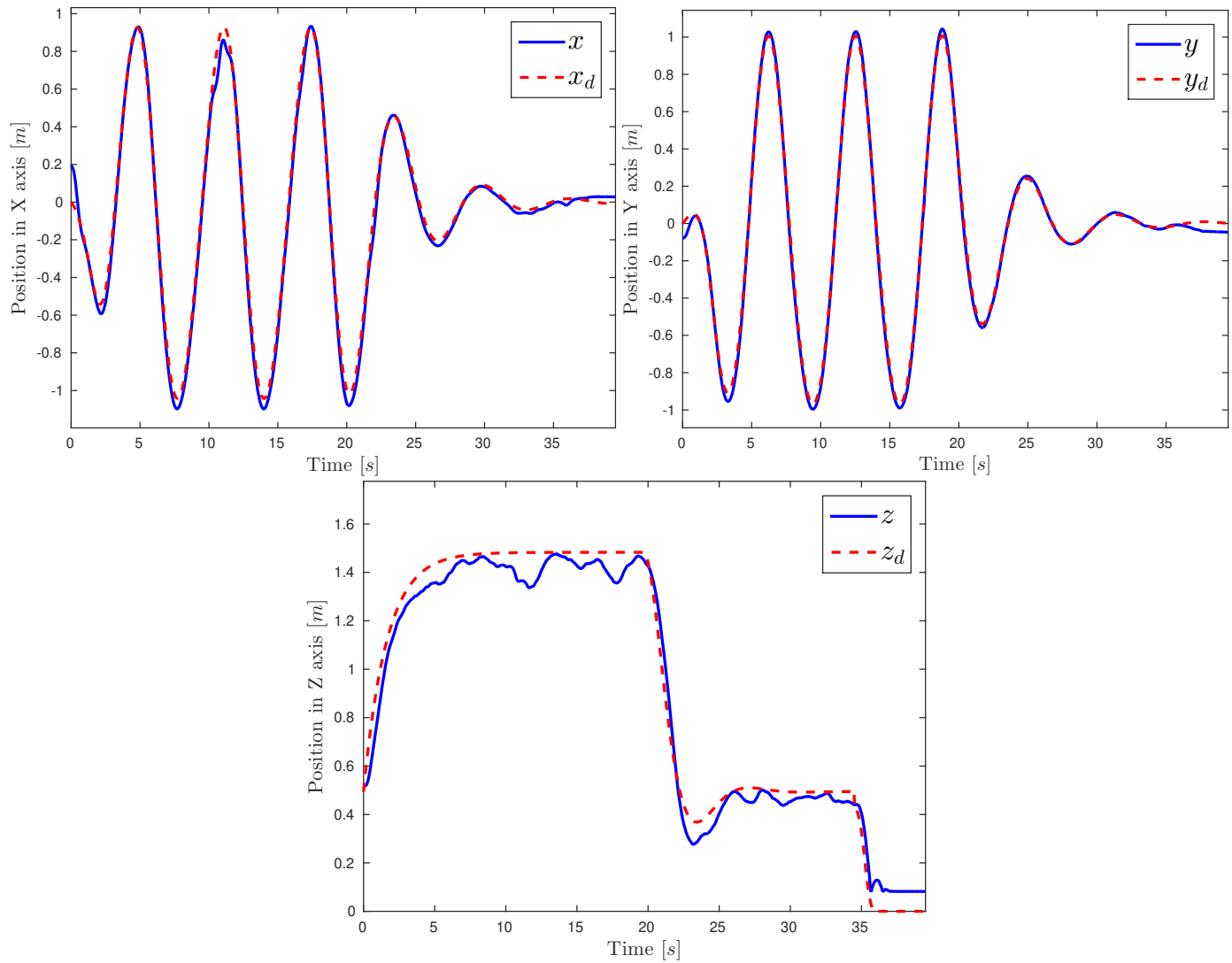

FIGURE 17 Quadrotor position response when following in real-time the desired trajectory.
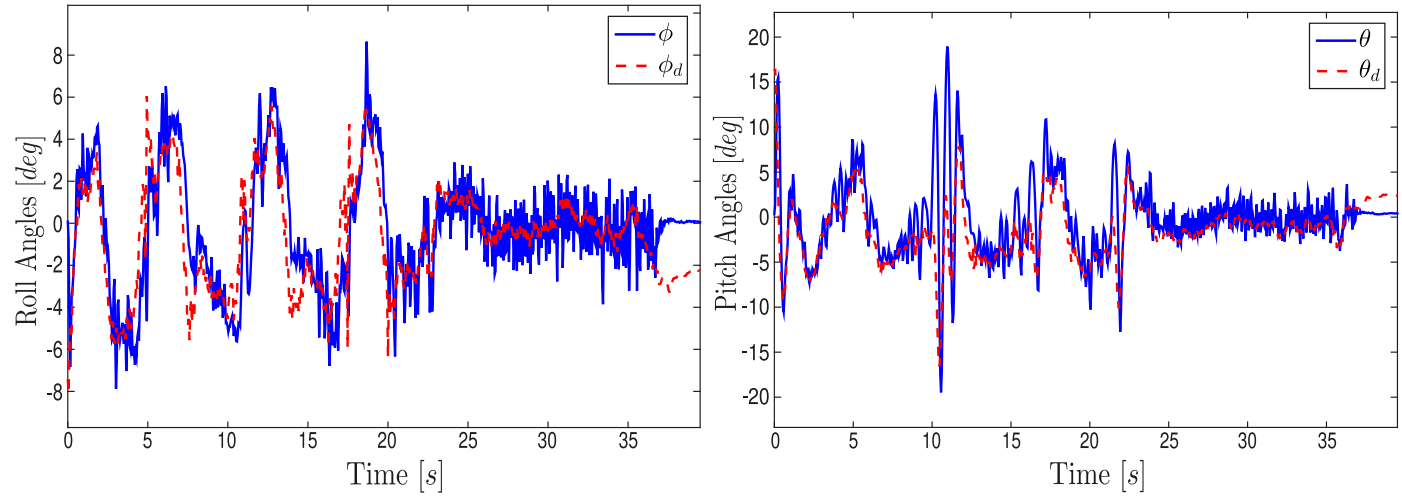

FIGURE 18 Attitude performance when the aerial vehicle follows autonomously the desired references. 
Figures 19 and 20 are used to illustrate the control inputs responses, $U_{1}$ is the controller responsible of achieving that each component $x, y$ and $z$ of the quadrotor converge to $x_{r}, y_{r}$ and $z_{r}$ respectively. Similarly, the control signal responses $U_{\phi}, U_{\theta}$ and $U_{\psi}$ ensure attitude convergence to $\phi \rightarrow \phi_{r}, \theta \rightarrow \theta_{r}$ and $\psi \rightarrow \psi_{r}=0$.

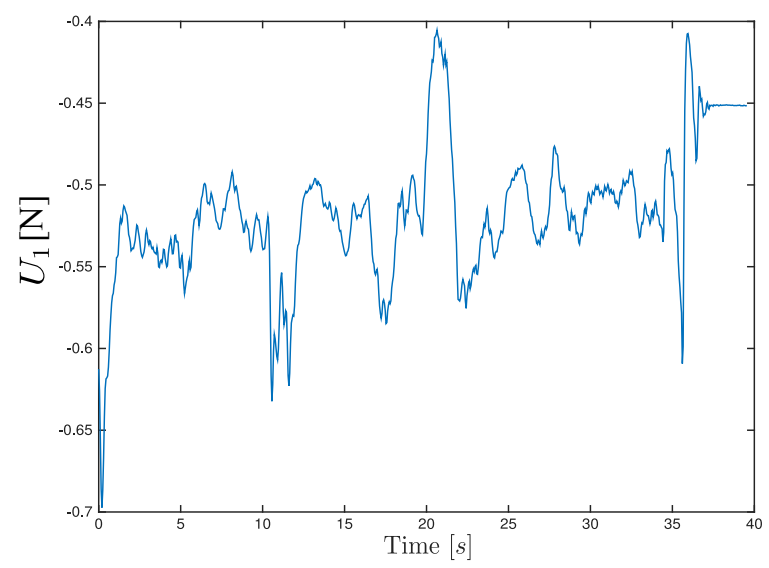

FIGURE 19 Main control performance when following the translational trajectory.

Note from Figure 20 that a chattering effect is displayed in the attitude control inputs due to the "sign" function which is present in the controllers. This chattering could be reduced by changing the "sign" functions by saturations, or a hyperbolic tangents, however, this would compromise the robustness of the system towards external disturbances.
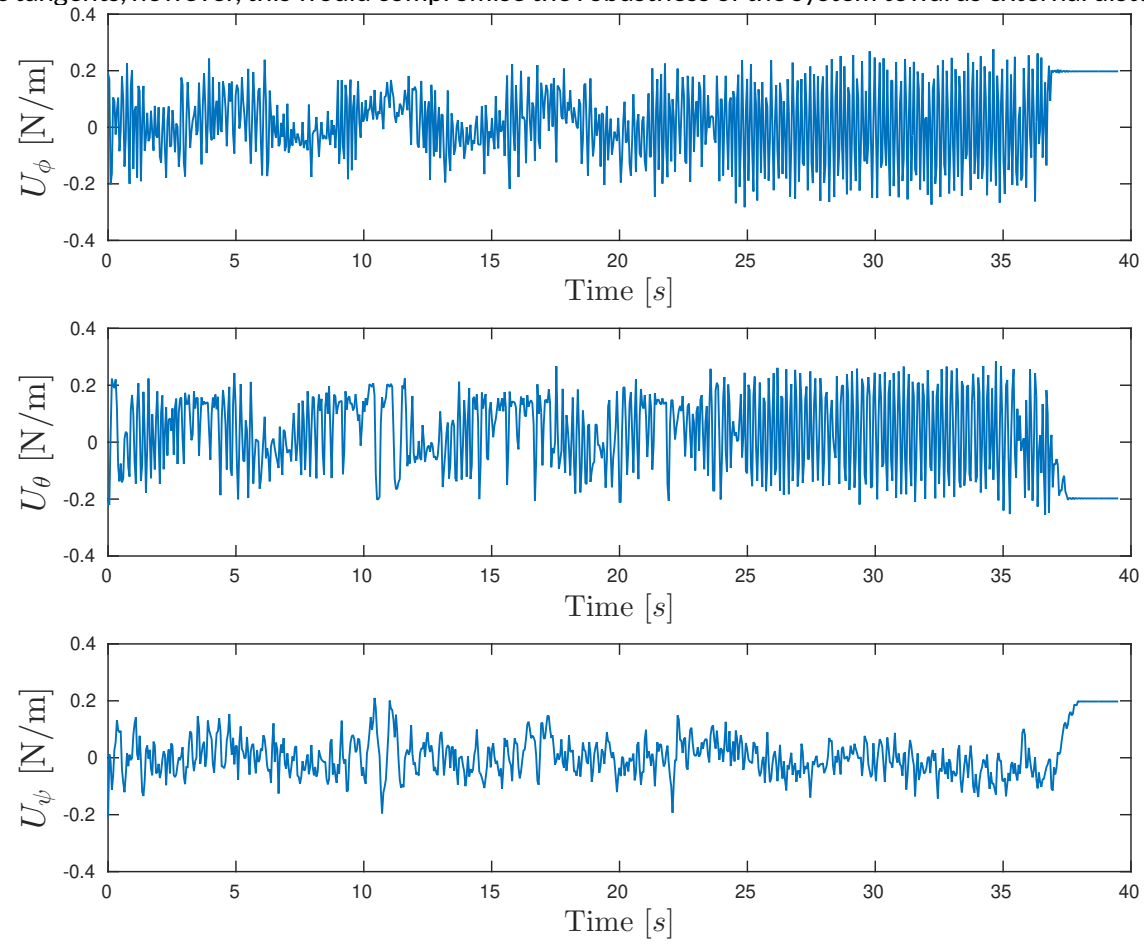

FIGURE 20 Control inputs responses for the attitude stabilization and tracking desired angles. 


\section{CONCLUSION}

In this paper, a nonlinear algorithm for tracking dynamic trajectories was proposed. The control scheme was obtained using a sliding mode approach which contains integral sliding properties. The algorithm was deduced step by step, and validated numerically and experimentally. The controller has robustness properties to compensate external and unknown disturbances or nonlinear uncertainties from the beginning time instant.

A dynamic trajectory was also proposed and conceived with properties for autonomous take-off, circular motion and landing for a quadrotor. The mathematical representation of the aerial robot was taken into account for designing the trajectory. The physical movements of the aerial vehicles were also taken into account to design the initial positions of the vehicle.

The proposed controller and the trajectory were validated in numerical simulations and also in flight tests. Results corroborate the good performance of the proposed trajectory-control scheme.

Since this work only tackled control design and validation problems, the topic of identifying external disturbances bounds was not addressed, future work will include the use of adaptive terminal sliding mode techniques, to identify uncertainties and disturbances.

\section{REFERENCES}

[1] Chen C. Linear System Theory and Design. CBS College Pub; 1984.

[2] Hespanha JP. Linear System Theory. Princeton University Press; 2009.

[3] Altug E, Ostrowski JP, Mahony R. Control of a quadrotor helicopter using visual feedback. In: Robotics and Automation, 2002. Proceedings. ICRA'02. IEEE International Conference on, vol. 1 IEEE; 2002. p. $72-77$.

[4] Bouabdallah S, Noth A, Siegwart R. PID vs LQ control techniques applied to an indoor micro quadrotor. In: Proc. of The IEEE International Conference on Intelligent Robots and Systems (IROS) IEEE; 2004. p. 2451-2456.

[5] Liu X, Gu G, Zhou K. Robust stabilization of MIMO nonlinear systems by backstepping. Automatica 1999;35(5):987-992.

[6] Yao B, Tomizuka M. Adaptive robust control of MIMO nonlinear systems in semi-strict feedback forms. Automatica 2001;37(9):1305-1321.

[7] Shaocheng T, Changying L, Yongming L. Fuzzy adaptive observer backstepping control for MIMO nonlinear systems. Fuzzy Sets and Systems 2009;160(19):2755-2775.

[8] Rigatos GG. Nonlinear control and filtering using differential flatness approaches: applications to electromechanical systems, vol. 25. Springer; 2015.

[9] Zerar M, Cazaurang F, Zolghadri A. Robust tracking of nonlinear MIMO uncertain flat systems. In: 2004 IEEE International Conference on Systems, Man and Cybernetics (IEEE Cat. No. 04CH37583), vol. 1 IEEE; 2004. p. 536-541.

[10] Victor S, Melchior P, Oustaloup A. Robust path tracking using flatness for fractional linear MIMO systems: a thermal application. Computers \& Mathematics with Applications 2010;59(5):1667-1678.

[11] Ledgerwood T, E M. Controllability and nonlinear control of rotational inverted pendulum. Advances in Robust and nonlinear Control Systems, ASME Journal on Dynamic Systems and Control 1992;43:81-88.

[12] Lukyanov AG. Optimal nonlinear block-control method. Procedings of the 2nd European control conference, Groningen, The Netherlands 1993;p. 1853-1855. 
[13] Lukyanov AG, Dodds SJ. Sliding mode block control of uncertain nonlinear plants. Procedings of the IFAC World Congress 1996;Section F-22B:241-246.

[14] Utkin V. Sliding Modes in Control and Optimization. Springer-Verlang; 1992.

[15] Utkin V, Guldner J, Shi J. Sliding Mode Control in Electromechanical Systems. Taylor and Francis; 1999.

[16] Young KD, Utkin VI, Ozguner U. A control engineer's guide to sliding mode control. In: Proceedings. 1996 IEEE International Workshop on Variable Structure Systems.-VSS'96- IEEE; 1996. p. 1-14.

[17] Zhihong M, YuXH. Terminal sliding mode control of MIMO linear systems. In: Proceedings of 35th IEEE conference on decision and control, vol. 4 IEEE; 1996. p. 4619-4624.

[18] Utkin V. Variable structure systems with sliding modes. IEEE Transactions on Automatic control 1977;22(2):212-222.

[19] Matthews GP, DeCarlo RA. Decentralized tracking for a class of interconnected nonlinear systems using variable structure control. Automatica 1988;24(2):187-193.

[20] Utkin V, Shi J. Integral sliding mode in systems operating under uncertainty conditions. vol. 4 IEEE; 1996. p. 4591 -4596.

[21] Benallegue A, Mokhtari A, Fridman L. High-order sliding-mode observer for a quadrotor UAV. International Journal of Robust and Nonlinear Control: IFAC-Affiliated Journal 2008;18(4-5):427-440.

[22] Shao X, Liu N, Liu J, Wang H. Model-assisted extended state observer and dynamic surface control-based trajectory tracking for quadrotors via output-feedback mechanism. International Journal of Robust and Nonlinear Control 2018;28(6):2404-2423.

[23] Shao X, Liu J, Cao H, Shen C, Wang H. Robust dynamic surface trajectory tracking control for a quadrotor UAV via extended state observer. International Journal of Robust and Nonlinear Control 2018;28(7):2700-2719.

[24] Dong Q, Zong Q, Tian B, Wang F. Adaptive-gain multivariable super-twisting sliding mode control for reentry RLV with torque perturbation. International Journal of Robust and Nonlinear Control 2017;27(4):620-638.

[25] Furtat I, Orlov Y, Fradkov A. Finite-time sliding mode stabilization using dirty differentiation and disturbance compensation. International Journal of Robust and Nonlinear Control 2019;29(3):793-809.

[26] Zou Y. Nonlinear robust adaptive hierarchical sliding mode control approach for quadrotors. International Journal of Robust and Nonlinear Control 2017;27(6):925-941.

[27] Tian B, Lu H, Zuo Z, Zong Q, Zhang Y. Multivariable finite-time output feedback trajectory tracking control of quadrotor helicopters. International Journal of Robust and Nonlinear Control 2018;28(1):281-295.

[28] Zhao B, Xian B, Zhang Y, Zhang X. Nonlinear robust sliding mode control of a quadrotor unmanned aerial vehicle based on immersion and invariance method. International Journal of Robust and Nonlinear Control 2015;25(18):3714-3731.

[29] Liu H, Li D, Xi J, Zhong Y. Robust attitude controller design for miniature quadrotors. International Journal of Robust and Nonlinear Control 2016;26(4):681-696.

[30] Dávila A, Moreno JA, Fridman L. Variable gains super-twisting algorithm: A Lyapunov based design; 2010. p. 968-973.

[31] Shtessel Y, Edwards C, Fridman L, Levant A. Sliding Mode Control and Observation. Birkhäuser; 2014.

[32] Jordan DW, Smith P. Nonlinear Ordinary Differential Equations. An introduction for Scientists and Engineers. Fourth ed. Oxford University Press; 2007.

[33] Qiao L, Zhang W. Adaptive non-singular integral terminal sliding mode tracking control for autonomous underwater vehicles. IET Control Theory \& Applications 2017;11(8):1293-1306.

[34] Qiao L, Zhang W. Double-loop integral terminal sliding mode tracking control for UUVs with adaptive dynamic compensation of uncertainties and disturbances. IEEE Journal of Oceanic Engineering 2019;44(1):29-53. 


\section{THEOREM PROOF}

Proof Considering that the exponential functions, $e^{\left(n\left(t-T_{F 1}\right)\right)} \in \tilde{f}(t)$ for $0 \leq t<T_{F 1}$ and $e^{\left(n\left(t-T_{F 2}\right)\right)} \in \bar{f}(t)$ for $0 \leq t<T_{F 2}$, are increasing functions with values inferior than one, then, it is easy to verify that if $n_{k}<n_{k+1}$

$$
\begin{aligned}
& 0<e^{\left(n_{k+1}\left(t-T_{F 1}\right)\right)}<e^{\left(n_{k}\left(t-T_{F 1}\right)\right)}<1, \\
& 0<e^{\left(n_{k+1}\left(t-T_{F 2}\right)\right)}<e^{\left(n_{k}\left(t-T_{F 2}\right)\right)}<1 .
\end{aligned}
$$

Define $T_{F 1} \leq T_{F 2}<T_{F}$, then $-T_{F 2} \leq-T_{F 1}$, which also implies that $t-T_{F 2} \leq t-T_{F 1}$ and taking $n>0 \Rightarrow n\left(t-T_{F 2}\right) \leq$ $n\left(t-T_{F 1}\right)$, as consequence $e^{\left(n\left(t-T_{F 2}\right)\right)} \leq e^{\left(n\left(t-T_{F 1}\right)\right)}$. Therefore for all $0 \leq t<T_{F_{2}}$,

$$
\bar{f}(t) \leqslant \tilde{f}(t)
$$

and also if $T_{F 1} \leq T_{F 2}$ and for all $T_{F 2} \leq t \leq T_{F}$ the following equality $\bar{f}(t)=\tilde{f}(t)=1$ is satisfied.
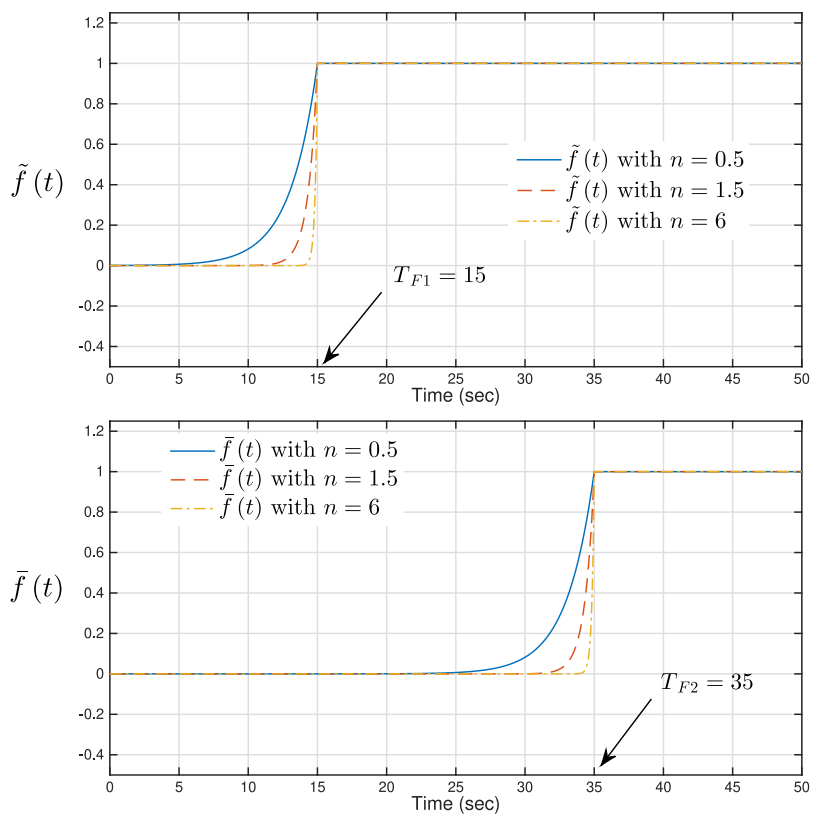

FIGURE $21 \tilde{f}(t)$ and $\bar{f}(t)$ analysis.

$\tilde{f}(t)$ and $\bar{f}(t)$ can be analyzed in Figure 21. Observe in this figure that when $n$ increases, $\tilde{f}(t)$ remains small and close to zero for almost the entire time interval $0 \leq t<T_{F 1}$, even when $t$ approaches $T_{F 1}$. From inequality $(54)$ also $\bar{f}(t) \approx 0$, therefore, it is possible to consider that, for all $0 \leq t<T_{F 1}, \tilde{g}(t)$ and $\bar{g}(t)$ becomes

$$
\tilde{g}(t)=1-\underbrace{\tilde{f}(t)}_{: \approx 0} \approx 1 \& \bar{g}(t)=1-\underbrace{\bar{f}(t)}_{: \approx 0} \approx 1 .
$$

The previous implies that for the time interval $0 \leq t<T_{F 1}$, the trajectory (14) is approximately equal to (12).Then it is possible to achieve circular take-off movements to a desired altitude $h$ as illustrated in Figure 3. 
Remember that the complete trajectory is defined for $T_{F 1} \leq T_{F 2}<T_{F}$, thus two cases are also studied:

Case 1: $T_{F 1}=T_{F 2}$.

Clearly $\tilde{f}(t)=\bar{f}(t)$, and for all $t \geq T_{F 1}$ it follows that $\tilde{f}(t)=\bar{f}(t)=1$. Therefore $\tilde{g}(t)$ and $\bar{g}(t)$ are zero. This implies that for all $T_{F 1} \leq t \leq T_{F}$, trajectory (15) is reduced to

$$
\begin{array}{lll}
\dot{x}_{1_{r}}=x_{2_{r}}, & \dot{y}_{1_{r}}=y_{2_{r}}, & \dot{z}_{1_{r}}=z_{2_{r},}, \\
\dot{x}_{2_{r}}=v_{x}, & \dot{y}_{2_{r}}=v_{y}, & \dot{z}_{2_{r}}=v_{z},
\end{array}
$$

where the initial conditions of the position and velocity are given by $x_{1_{r}}\left(T_{F_{1}}\right), y_{1_{r}}\left(T_{F_{1}}\right), z_{1_{r}}\left(T_{F_{1}}\right)$ and $x_{2_{r}}\left(T_{F_{1}}\right), y_{2_{r}}\left(T_{F_{1}}\right)$, $z_{2_{r}}\left(T_{F_{1}}\right)$. Then obtaining the derivatives of $\sigma_{x}, \sigma_{y}$ and $\sigma_{z}$ given in (17) results in

$$
\begin{aligned}
& \dot{\sigma}_{x}=v_{x}+\left(3 x_{1_{r}}^{2}+y_{1_{r}}^{2}-\mu_{2}\right) x_{2_{r}}+\left(2 x_{1_{r}} y_{1_{r}}-\gamma_{2}\right) y_{2_{r}}, \\
& \dot{\sigma}_{y}=v_{y}+\left(2 x_{1_{r}} y_{1_{r}}+\gamma_{2}\right) x_{2 r}+\left(3 y_{1_{r}}^{2}+x_{1_{r}}^{2}-\mu_{2}\right) y_{2_{r}}, \\
& \dot{\sigma}_{z}=v_{z}+\bar{k} z_{2_{r}} .
\end{aligned}
$$

Substituting $v_{x}, v_{y}$ and $v_{z}$ from (16) into the above, it yields

$$
\dot{\sigma}_{x}=-\bar{k}_{x} \sigma_{x}, \quad \dot{\sigma}_{y}=-\bar{k}_{y} \sigma_{y}, \quad \dot{\sigma}_{z}=-\bar{k}_{z} \sigma_{z}
$$

with positive gains $\bar{k}_{x}, \bar{k}_{y}$ and $\bar{k}_{z}$. The asymptotic solutions of $(56)$ are given by $\sigma_{x}(t)=e^{-\bar{k}_{x}\left(t-T_{F 1}\right)} \sigma_{x}\left(T_{F 1}\right), \sigma_{y}(t)=$ $e^{-\bar{k}_{y}\left(t-T_{F 1}\right)} \sigma_{y}\left(T_{F 1}\right)$ and $\sigma_{z}(t)=e^{-\bar{k}_{z}\left(t-T_{F 1}\right)} \sigma_{z}\left(T_{F 1}\right)$ for all $T_{F_{1}} \leq t \leq T_{F}$, see Figure 22.
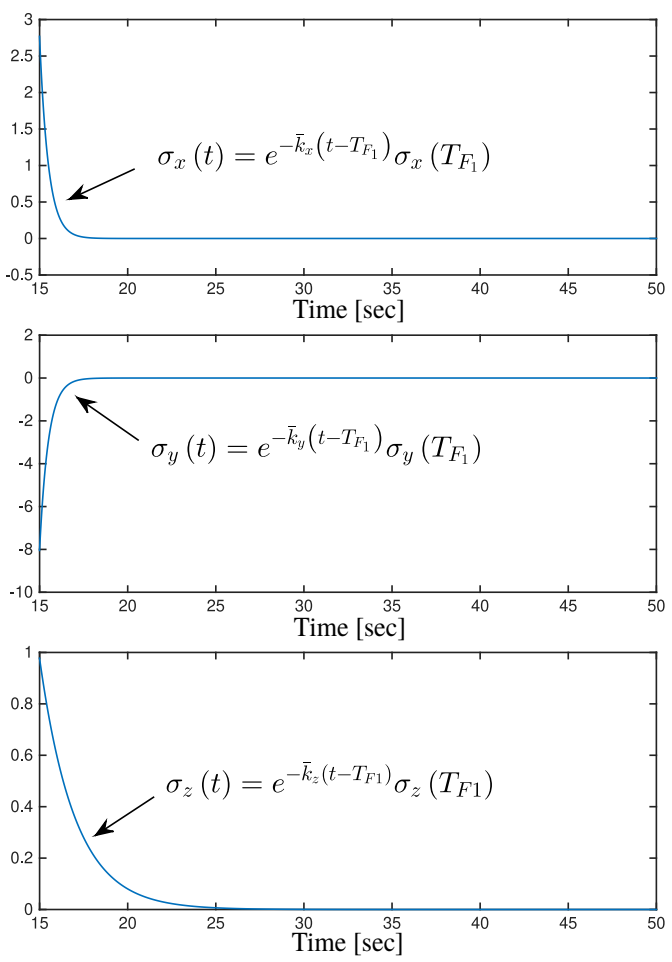

FIGURE $22 \sigma_{i}$ performance in the time interval $T_{F_{1}} \leq t \leq T_{F}$. 
The previous implies that for all $T_{F_{1}} \leq t \leq T_{F}, \dot{x}_{1_{r}}, \dot{y}_{1_{r}}$ and $\dot{z}_{1_{r}}$ are given by

$$
\begin{aligned}
& \dot{x}_{1_{r}}=\mu_{2} x_{1_{r}}+\gamma_{2} y_{1_{r}}-x_{1_{r}}\left(x_{1_{r}}^{2}+y_{1_{r}}^{2}\right)+e^{-\bar{k}_{x}\left(t-T_{F 1}\right)} \sigma_{x}\left(T_{F 1}\right), \\
& \dot{y}_{1_{r}}=-\gamma_{2} x_{1_{r}}+\mu_{2} y_{1_{r}}-y_{1_{r}}\left(x_{1_{r}}^{2}+y_{1_{r}}^{2}\right)+e^{-\bar{k}_{y}\left(t-T_{F 1}\right)} \sigma_{y}\left(T_{F 1}\right), \\
& \dot{z}_{1_{r}}=-\bar{k}\left(z_{1_{r}}-z_{1_{r}}(0)\right)+e^{-\bar{k}_{z}\left(t-T_{F 1}\right)} \sigma_{z}\left(T_{F 1}\right),
\end{aligned}
$$

with $\mu_{2} \leq 0$ and $\left(\gamma_{2}>0\right.$ or $\left.\gamma_{2}<0\right)$.
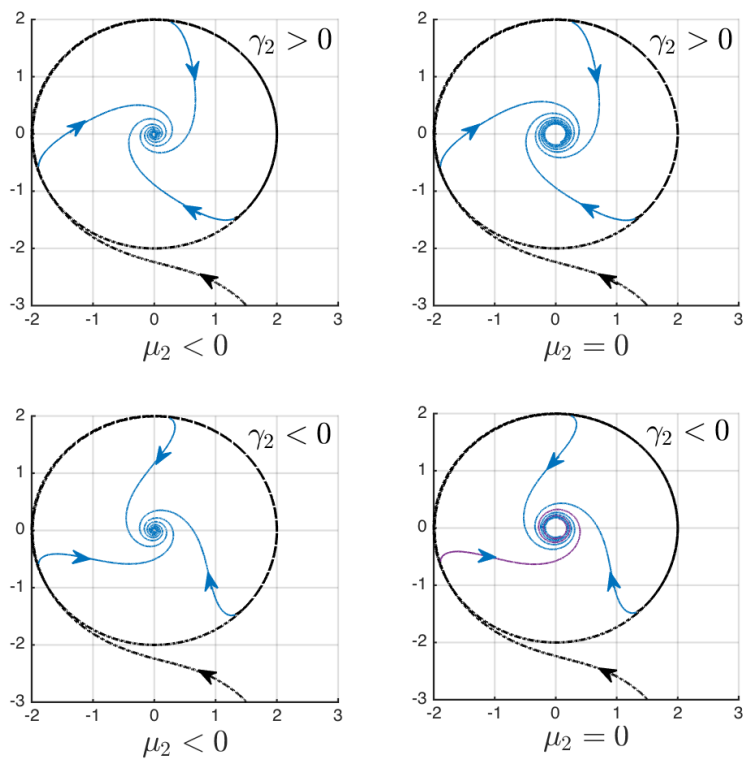

FIGURE 23 Bifurcation cases for the interval $T_{F 1} \leq t \leq T_{F}$.

Notice that $v_{x}$ and $v_{y}$ were designed such that $\sigma_{x}, \sigma_{y} \rightarrow 0$ asymptotically, implying that $\left(x_{1_{r}}, y_{1_{r}}\right) \rightarrow(0,0)$ in a spiral motion according to the convergence cases with $T_{F 1}=15,17$ and 19 seconds given in Figure 23. Similarly $v_{z}$ was designed to $\sigma_{z} \rightarrow 0$ such that $z_{1_{r}} \rightarrow z_{1_{r}}(0)$ asymptotically, this means that $v_{x}, v_{y}$ and $v_{z}$ are necessary for achieving $\left(x_{1}, y_{1_{r}}, z_{1_{r}}\right) \rightarrow\left(0,0, z_{1_{r}}(0)\right)$ in the time interval $T_{F_{1}} \leq t \leq T_{F}$, and thus producing landing. The previous is illustrated in Figure 5 , where the landing trajectory to $\left(0,0, z_{1_{r}}(0)\right)$ is presented as a solid (blue) line.

With Case1, the landing stage of movement is obtained such that, together with the first stage of movement, the first scenario has been completed.

Case 2: $T_{F 1}<T_{F 2}$.

The trajectory is defined in the interval $T_{F 1} \leq T_{F 2}<T_{F}$, and $\forall 0 \leq t<T_{F 2}$, then $\bar{f}(t) \leqslant \tilde{f}(t)$. From Case 1 , if $T_{F 1}=T_{F 2}$ then $\bar{f}(t)=\tilde{f}(t)$. Therefore the only possibility for the inequality $T_{F 1}<T_{F 2}$ is that $\bar{f}(t)<\tilde{f}(t) \forall 0 \leq t<T_{F 2}$. Observe that $\forall T_{F 1} \leq t<T_{F 2}$, thus $\tilde{f}(t)=1$. In this way $\bar{f}(t)<1$ and $\bar{f}(t) \approx 0$ even when $t$ is close to $T_{F 2}$. Then $\forall T_{F 1} \leq t<T_{F 2}$ is possible consider that $\tilde{g}(t)=0$ and $\bar{g}(t)=1$. 
Therefore, for $T_{F 1} \leq t<T_{F 2}$ the trajectory (15) is approximately equal to

$$
\begin{aligned}
& \dot{x}_{1_{r}}=x_{2_{r}}, \quad \dot{y}_{1_{r}}=y_{2_{r}}, \quad \dot{z}_{1_{r}}=z_{2_{r}}, \\
& \dot{x}_{2_{r}}=v_{x}, \quad \dot{y}_{2_{r}}=v_{y}, \quad \dot{z}_{2_{r}} \approx u_{z} .
\end{aligned}
$$

Similarly the initial conditions of the position and velocity are given by $x_{1_{r}}\left(T_{F_{1}}\right), y_{1_{r}}\left(T_{F_{1}}\right), z_{1_{r}}\left(T_{F_{1}}\right)$ and $x_{2_{r}}\left(T_{F_{1}}\right)$, $y_{2_{r}}\left(T_{F_{1}}\right), z_{2_{r}}\left(T_{F_{1}}\right)$. The derivatives for $\sigma_{x}$ and $\sigma_{y}$ were previously obtained, the changing value in this case is the altitude, thus

$$
\dot{S}_{z}=u_{z}+\bar{k} z_{2 r}
$$

This implies that

$$
\dot{\sigma}_{x}=-\bar{k}_{x} \sigma_{x}, \quad \dot{\sigma}_{y}=-\bar{k}_{y} \sigma_{y}, \quad \dot{S}_{z}=-k_{z} S_{z}
$$

with positive gains $\bar{k}_{x}, \bar{k}_{y}$ and $k_{z}$. The asymptotic solutions of $(56)$ are given by $\sigma_{x}(t)=e^{-\bar{k}_{x}\left(t-T_{F 1}\right)} \sigma_{x}\left(T_{F 1}\right), \sigma_{y}(t)=$ $e^{-\bar{k}_{y}\left(t-T_{F 1}\right)} \sigma_{y}\left(T_{F 1}\right)$ and $S_{z}(t)=e^{-k_{z}\left(t-T_{F 1}\right)} S_{z}\left(T_{F 1}\right)$ for all $T_{F 1} \leq t<T_{F 2}$, graphically it can be seen in Figure 24 .
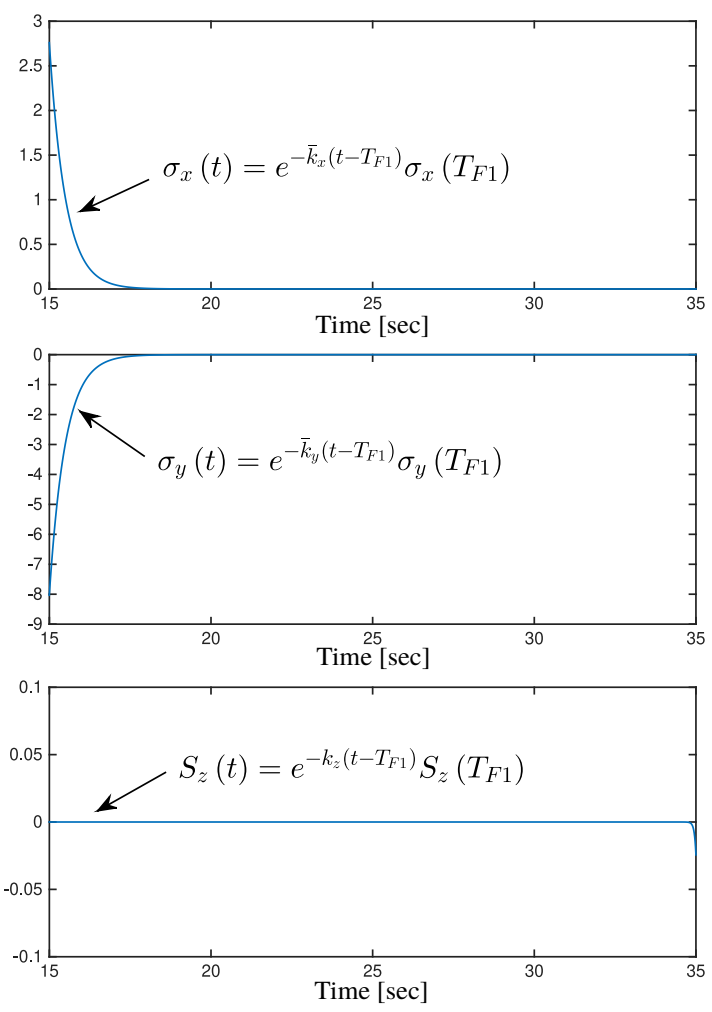

FIGURE $24 \sigma_{x}, \sigma_{y}$ and $S_{z}$ behavior in the interval time $T_{F 1} \leq t<T_{F 2}$. 
The previous implies that $\dot{x}_{1_{r}}, \dot{y}_{1_{r}}$ and $\dot{z}_{1_{r}}$ in the time interval $T_{F 1} \leq t<T_{F 2}$ are given by

$$
\begin{aligned}
& \dot{x}_{1_{r}}=\mu_{2} x_{1_{r}}+\gamma_{2} y_{1_{r}}-x_{1_{r}}\left(x_{1_{r}}^{2}+y_{1_{r}}^{2}\right)+e^{-\bar{k}_{x}\left(t-T_{F 1}\right)} \sigma_{x}\left(T_{F 1}\right), \\
& \dot{y}_{1_{r}}=-\gamma_{2} x_{1_{r}}+\mu_{2} y_{1_{r}}-y_{1_{r}}\left(x_{1_{r}}^{2}+y_{1_{r}}^{2}\right)+e^{-\bar{k}_{y}\left(t-T_{F 1}\right)} \sigma_{y}\left(T_{F 1}\right), \\
& \dot{z}_{1_{r}}=-k\left(z_{1_{r}}-h\right)+e^{-k_{z}\left(t-T_{F 1}\right)} S_{z}\left(T_{F 1}\right),
\end{aligned}
$$

with $\mu_{2} \leq 0$ and $\left(\gamma_{2}>0\right.$ or $\left.\gamma_{2}<0\right)$.

Notice that $v_{x}$ and $v_{y}$ produce that $\sigma_{x}, \sigma_{y} \rightarrow 0$ asymptotically, in consequence $\dot{x}_{1_{r}} \rightarrow \mu_{2} x_{1_{r}}+\gamma_{2} y_{1_{r}}-x_{1_{r}}\left(x_{1_{r}}^{2}+y_{1_{r}}^{2}\right)$, $\dot{y}_{1_{r}} \rightarrow-\gamma_{2} x_{1_{r}}+\mu_{2} y_{1_{r}}-y_{1_{r}}\left(x_{1_{r}}^{2}+y_{1_{r}}^{2}\right)$, implying that $\left(x_{1_{r}}, y_{1_{r}}\right) \rightarrow(0,0)$ in a spiral motion. $u_{z}$ makes that $S_{z} \rightarrow 0, \dot{z}_{1_{r}} \rightarrow$ $-k\left(z_{1_{r}}-h\right)$, and $z_{1_{r}} \rightarrow h$ asymptotically. Therefore $v_{x}, v_{y}$ and $u_{z}$ are necessary for achieving $\left(x_{1_{r}}, y_{1_{r}}, z_{1_{r}}\right) \rightarrow(0,0, h)$ in the interval time $T_{F 1} \leq t<T_{F 2}$, and producing the spiral movement around $(0,0, h)$. This is illustrated in solid (blue) line in the Figure 25.

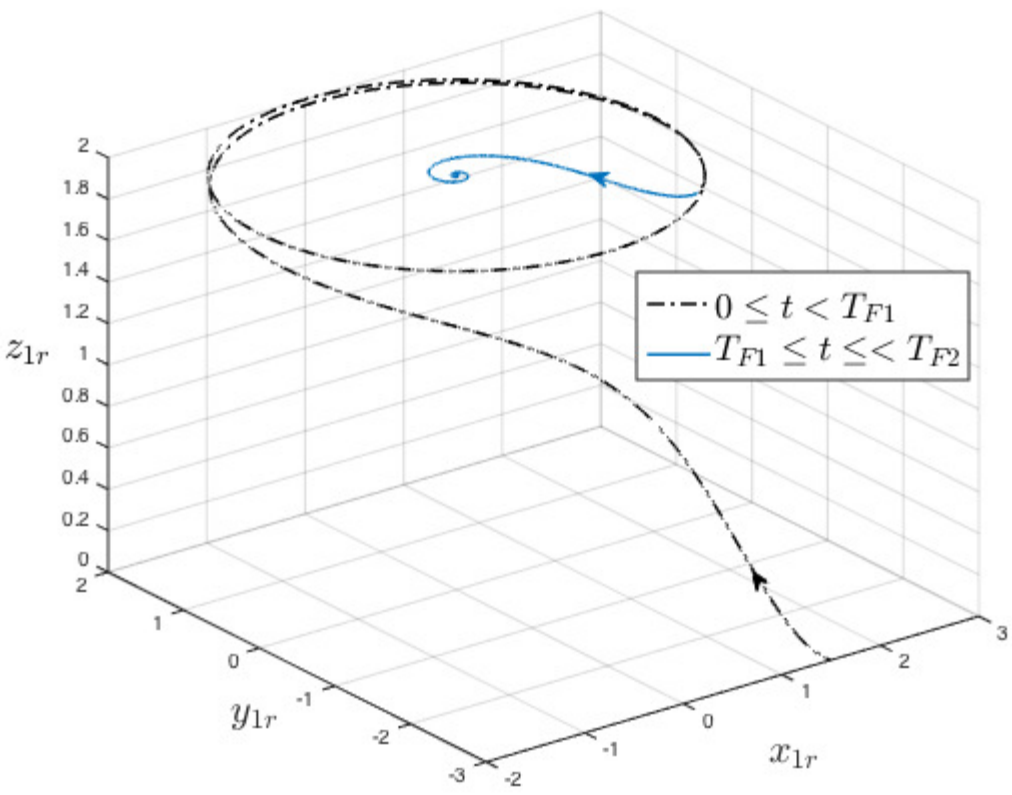

FIG URE 25 Circular take-off trajectory with spiral motion around $(0,0, h)$.

Once proved that, for all $0 \leq t<T_{F 2}$ and considering $T_{F 1}<T_{F 2}$, the proposed trajectory (15) is valid for take-off and circular movement stages at a desired altitude $h$. The final step is then to prove that for all $T_{F 2} \leq t \leq T_{F}$, (15) produces also a landing trajectory. Notice that $\bar{f}(t)=\tilde{f}(t)=1$ for all $T_{F 2} \leq t \leq T_{F}$, then $\tilde{g}(t)$ and $\bar{g}(t)$ are zero. This implies that system becomes as (55) with initial position and velocity conditions given by $x_{1_{r}}\left(T_{F 2}\right), y_{1_{r}}\left(T_{F 2}\right), z_{1_{r}}\left(T_{F 2}\right)$ and $x_{2_{r}}\left(T_{F 2}\right), y_{2_{r}}\left(T_{F 2}\right), z_{2_{r}}\left(T_{F 2}\right)$.

The asymptotic solutions of are given by $\sigma_{x}(t)=e^{-\bar{k}_{x}\left(t-T_{F 2}\right)} \sigma_{x}\left(T_{F 2}\right), \sigma_{y}(t)=e^{-\bar{k}_{y}\left(t-T_{F 2}\right)} \sigma_{y}\left(T_{F 2}\right)$ and $\sigma_{z}(t)=$ $e^{-\bar{k}_{z}\left(t-T_{F 2}\right)} \sigma_{z}\left(T_{F 2}\right)$ for all $T_{F 2} \leq t \leq T_{F}$, the performance of these solutions can be seen in Figure 26. 

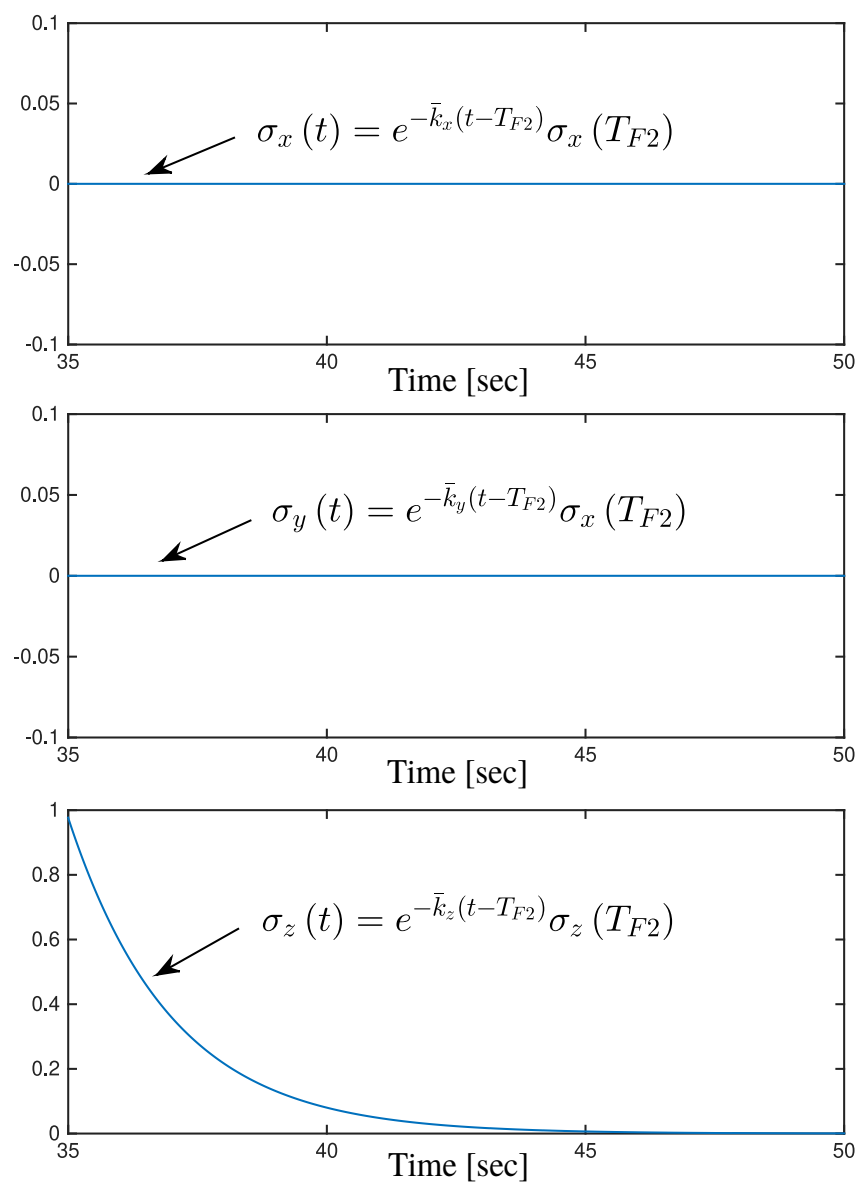

FIGURE $26 \sigma_{x}, \sigma_{y}$ and $\sigma_{z}$ behavior in the interval time $T_{F 2} \leq t \leq T_{F}$

All the above implies that $\dot{x}_{1_{r}}, \dot{y}_{1_{r}}$ and $\dot{z}_{1_{r}}$ in the interval time $T_{F 2} \leq t \leq T_{F}$ are given by

$$
\begin{aligned}
& \dot{x}_{1_{r}}=\mu_{2} x_{1_{r}}+\gamma_{2} y_{1_{r}}-x_{1_{r}}\left(x_{1_{r}}^{2}+y_{1_{r}}^{2}\right)+e^{-\bar{k}_{x}\left(t-T_{F 2}\right)} \sigma_{X}\left(T_{F 2}\right) \\
& \dot{y}_{1_{r}}=-\gamma_{2} x_{1_{r}}+\mu_{2} y_{1_{r}}-y_{1_{r}}\left(x_{1_{r}}^{2}+y_{1_{r}}^{2}\right)+e^{-\bar{k}_{y}\left(t-T_{F 2}\right)} \sigma_{y}\left(T_{F 2}\right) \\
& \dot{z}_{1_{r}}=-\bar{k}\left(z_{1_{r}}-z_{1_{r}}(0)\right)+e^{-\bar{k}_{z}\left(t-T_{F 2}\right)} \sigma_{z}\left(T_{F 2}\right)
\end{aligned}
$$

with $\mu_{2} \leq 0$ and $\left(\gamma_{2}>0\right.$ or $\left.\gamma_{2}<0\right)$.

Finally with the development of Case 2 , two stages of movement (spiral motion and landing to a desired point) are obtained, which together with the first stage of movement (circular take-off to a desired altitude $h$ ), the second scenario has been completed. 